\title{
A combinatoric shortcut to evaluate $\mathrm{CHY}$-forms
}

\author{
Tianheng Wang, ${ }^{a}$ Gang Chen, ${ }^{b, c, 1}$ Yeuk-Kwan E. Cheung ${ }^{a}$ and Feng $\mathbf{X} \mathbf{u}^{d}$ \\ ${ }^{a}$ Department of Physics, Nanjing University, \\ Nanjing, Jiangsu Province, China \\ ${ }^{b}$ Department of Physics, Zhejiang Normal University, \\ Jinhua, Zhejiang Province, China \\ ${ }^{c}$ Department of Physics and Astronomy, Uppsala University, \\ Uppsala, Sweden \\ ${ }^{d}$ Weavi Corporation Limited, \\ Nanjing, Jiangsu Province, China \\ E-mail: tianhengwang@outlook.com, gang.chen@physics.uu.se, \\ cheung@nju.edu.cn, schyfeng@gmail.com
}

ABSTRACT: In our recent work, we proposed a differential operator for the evaluation of the multi-dimensional residues on isolated (zero-dimensional) poles. In this paper we discuss some new insight on evaluating the (generalized) Cachazo-He-Yuan (CHY) forms of the scattering amplitudes using this differential operator. We introduce a tableau representation for the coefficients appearing in the proposed differential operator. Combining the tableaux with the polynomial form of the scattering equations, the evaluation of the generalized CHY form becomes a simple combinatoric problem. It is thus possible to obtain the coefficients arising in the differential operator in a straightforward way. We present the procedure for a complete solution of the $n$-gon amplitudes at one-loop level in a generalized CHY form. We also apply our method to fully evaluate the one-loop five-point amplitude in the maximally supersymmetric Yang-Mills theory; the final result is identical to the one obtained by Q-Cut.

Keywords: Scattering Amplitudes, Differential and Algebraic Geometry, Extended Supersymmetry

ARXiv EPRINT: 1701.06488

\footnotetext{
${ }^{1}$ Corresponding author.
} 


\section{Contents}

1 Introduction 1

2 Prescription for determining differential operators 3

2.1 Toy model: one-loop four-point SYM integrand 3

2.2 Preliminary: notations \& tableaux $\quad 6$

2.3 Coefficient generating algorithm 9

3 One-loop five-point SYM amplitude $\quad \mathbf{1 3}$

3.1 Simplify the one-loop generalized CHY integrand 13

$\begin{array}{ll}3.2 & \text { Transformations to the prepared CHY integrand } \\ \end{array}$

$\begin{array}{ll}3.3 & \text { Evaluating the prepared form of the five point integrand } \\ \end{array}$

$\begin{array}{ll}3.4 & \text { Analysis for the spurious poles } \\ \end{array}$

4 One-loop $n$-gon amplitude: a direct evaluation 22

5 Conclusion and outlook $\quad 24$

\section{Introduction}

Scattering equations are derived at tree level for the high-energy behavior of string theory in $[1,2]$ and have drawn attention from theoretical physicists in diversified contexts $[3,4]$. Incorporating scattering equations, Cachazo, He and Yuan [5-7] propose a closed formula for arbitrary $n$-point tree amplitudes in a variety of massless quantum field theories. This form is proven by Dolan and Goddard in [8] for Yang-Mills theory in arbitrary dimensions and a polynomial form of the scattering equations is obtained by the same group in [9].

The scattering equations are generalized to loop levels in a number of contexts, such as open string theory, pure spinor formalism of superstring, and ambitwistor string theory [1012]. The CHY expressions are subsequently extended to one and two loops for the bi-adjoint scalar theory, gauge theory and gravity in [13-16]. In addition to the ambitwitor approach, the one-loop generalized CHY forms are obtained from tree-level ones in one higher spatial dimension in $[17,18]$ for scalar and gauge theories, and higher-loop CHY forms for scalar theory are constructed in [19].

Besides constructing the generalized $\mathrm{CHY}$ forms, it is also a challenge to evaluate such multi-dimensional CHY integrals. Indirect methods, such as "building block method" [2023] and "Integration rules" [24-31], evaluate the generalized CHY integrals reductively. Direct approaches are also explored. At tree level, the $n$-point $\mathrm{CHY}$ form is evaluated for gauge and gravity theories in special kinematics in [32] and the scattering equations are solved in four dimensions up to six points in [33-35]. Elimination theory is exploited in 
solving the scattering equations up to seven points in [36, 37] and a general prescription based on elimination theory is proposed in [38]. A direct evaluation of the CHY form for the MHV tree amplitude is given in [39]. Algebraic geometry based methods - the companion matrix method [40], Bezoutian matrix method [41], and polynomial reduction techniques $[42,43]$ - are employed to evaluate the CHY expressions, without solving the underlying scattering equations.

In our previous work [44] we proposed a conjecture that enables us to compute multidimensional residues on isolated (zero-dimensional) poles by a differential operator. Here we briefly recall that conjecture.

Suppose $f_{1}, f_{2}, \ldots, f_{k}$ are homogeneous polynomials in complex variables $z_{1}, z_{2}, \ldots z_{k}$ of degrees $d_{1}, d_{2}, \ldots, d_{k}$ respectively. And we assume that the common zeros of $f_{1}, f_{2}$, $\ldots, f_{k}$ consist of a single isolated point $p$. Let $\mathcal{R}\left(z_{i}\right)$ be a holomorphic function in a neighborhood of $p$. Then the conjecture states that the residue of $\mathcal{R}$ at $p$ can be computed by a differential operator $\mathbb{D}$ as follows,

$$
\operatorname{Res}_{\left\{\left(f_{1}\right), \cdots,\left(f_{k}\right)\right\}, p}[\mathcal{R}] \equiv \oint \frac{d z_{1} \wedge \cdots \wedge d z_{k}}{f_{1} \cdots f_{k}} \mathcal{R}=\left.\mathbb{D}[\mathcal{R}]\right|_{z_{i} \rightarrow 0}
$$

where $\mathbb{D}$ is of the form

$$
\mathbb{D}=\sum_{\left\{r_{i}\right\}} a_{r_{1}, r_{2}, \cdots, r_{k}}\left(\partial_{1}\right)^{r_{1}}\left(\partial_{2}\right)^{r_{2}} \cdots\left(\partial_{k}\right)^{r_{k}}
$$

Here coefficients $a_{r_{1}, r_{2}, \ldots, r_{k}}$ are $z$-independent constants and $\partial_{i}=\frac{\partial}{\partial z_{i}}, i=1, \ldots, k$, where $r_{i}$ 's are non-negative integers. The sum is done over all solutions of the equation $\sum_{i=1}^{k} r_{i}=$ $\sum_{h=1}^{k} d_{h}-k$. Moreover, it is conjectured that $\mathbb{D}$ is uniquely determined by two conditions respectively from 1) the local duality theorem $[45,46]$ and 2) the intersection number of the divisors $D_{i}=\left(f_{i}\right)$.

This conjecture is verified numerically to be widely applicable for computing both degenerate and non-degenerate multi-dimensional residues, as long as the poles are isolated. In the application to evaluating the generalized $\mathrm{CHY}$ integrals, the linear equations for $a_{r_{1}, \cdots, r_{k}}$ arising from the local duality theorem and the intersection number requirement are the structures of interest. In this paper, we study these structures by introducing a tableau representation for each $a_{r_{1}, \cdots, r_{k}}$. Using the tableau representation, we explain how to obtain the differential operator efficiently, especially for the polynomial scattering equations, which makes the evaluation of the generalized CHY forms a simple combinatoric problem. In this way, the evaluation of CHY integrals in their prepared forms (which we define later in this paper) is easily achieved for any number of external lines at one loop.

This leads straightforwardly to a full evaluation of the $n$-gon one-loop integrand in the generalized CHY form, since the $n$-gon integrand is naturally of the prepared form. Furthermore, for the amplitudes in super-Yang-Mills, the one-loop integrands for four and five particles can also be recast into the prepared forms using the cross-ratio identites derived in [31], which are then calculated effortlessly. We expect that one-loop integrands for higher points can also be transformed into the prepared forms or similar expressions. Upon evaluating the prepared forms, the final result is identical to the Q-cut representation of the expression obtained in [47], while the Q-cut method is proposed in [48]. 


\section{Prescription for determining differential operators}

In this section we introduce our new method to determine the differential operators that is more efficient than the approach taken in [44]. We begin with a warmup example for the four point one loop SYM integrand. After that we present the notations used in obtaining the coefficients in the differential operators. Finally we use this method to get the complete solutions of the coefficients for a particular class of CHY integrands.

\subsection{Toy model: one-loop four-point SYM integrand}

The one-loop scattering amplitude for four external gluons in $\mathcal{N}=4 \mathrm{SYM}$ has been revisited in various contexts in literature $[47,49]$. The generalized CHY integral for its loop integrand is given in $[14,15]$. A detailed analysis of the evaluation of this generalized CHY integral has already been presented in [44]. In this section we reconsider this example as a toy model to illustrate the main ideas of the method proposed in this paper. Details of and more general discussions on the method are to appear in later sections.

Firstly let us recall the settings. The four-point generalized CHY form of the integrand obtained in $[14,15]$ reads,

$$
\mathcal{I}_{4}=\frac{\langle 12\rangle^{4}}{\langle 12\rangle\langle 23\rangle\langle 34\rangle\langle 45\rangle\langle 51\rangle} \oint \frac{d \sigma_{1} \cdots d \sigma_{3}}{h_{1} \cdots h_{3}} \mathrm{PT}_{4} \prod_{i<j}^{4}\left(\sigma_{i}-\sigma_{j}\right),
$$

where $\mathrm{PT}_{4}$ is the well-known Parke-Taylor factor that reads

$$
\mathrm{PT}_{4}=\sum_{\rho \in \mathcal{S}_{4}} \frac{1}{\sigma_{\rho(1)}\left(\sigma_{\rho(1)}-\sigma_{\rho(2)}\right)\left(\sigma_{\rho(2)}-\sigma_{\rho(3)}\right)\left(\sigma_{\rho(3)}-\sigma_{\rho(4)}\right)},
$$

where $\mathcal{S}_{4}$ is the cyclic permutation group of four objects. For illustrative purpose, here we only consider the first term in $\mathrm{PT}_{4}$ which leads to the following integral,

$$
\mathcal{I}_{4}^{1}=\frac{\langle 12\rangle^{4}}{\langle 12\rangle\langle 23\rangle\langle 34\rangle\langle 45\rangle\langle 51\rangle} \oint \frac{d \sigma_{1} \wedge \cdots \wedge d \sigma_{3}}{h_{1} \cdots h_{3}} \frac{\left(\sigma_{3}\right)\left(\sigma_{2}-\sigma_{4}\right)}{\sigma_{1}},
$$

where we fix the gauge $\sigma_{4}=1$ and the polynomials $h_{i}$ 's are

$$
h_{1}=\sum_{i=1}^{4} \ell_{i} \sigma_{i}, \quad h_{2}=(-) \sum_{i_{1}<i_{2}}^{4} \sigma_{i_{1}} \sigma_{i_{2}} \ell_{i_{1} i_{2}}, \quad h_{3}=\sum_{i_{1}<i_{2}<i_{3}}^{4} \sigma_{i_{1} \cdots i_{3}} \ell_{i_{1} \cdots i_{3}}
$$

and we denote the loop momentum as $\ell$ and have used the following notations,

$\sigma_{i_{1} \cdots i_{m}} \equiv \prod_{r=1}^{m} \sigma_{r}, \quad \ell_{i} \equiv \ell \cdot k_{i}, \quad \ell_{i_{1} \cdots i_{m}} \equiv\left(\ell \cdot k_{i_{1} \cdots i_{m}}-\frac{1}{2} k_{i_{1} \cdots i_{m}}^{2}\right), \quad k_{i_{1} \cdots i_{m}} \equiv \sum_{r=1}^{m} k_{i_{r}}$.

As shown in [44], using the global residue theorem, the integral can be rewritten as the following

$$
\mathcal{I}_{4}^{1}=\frac{-\langle 12\rangle^{4}}{\langle 12\rangle\langle 23\rangle\langle 34\rangle\langle 45\rangle\langle 51\rangle} \oint \frac{d \sigma_{1} \wedge \cdots \wedge d \sigma_{3} \wedge d \sigma_{4}^{\prime}}{\tilde{h}_{1} \cdots \tilde{h}_{3} \sigma_{1}} \frac{\sigma_{3}\left(\sigma_{2}-\sigma_{4}^{\prime}\right)}{\sigma_{4}^{\prime}-1}
$$




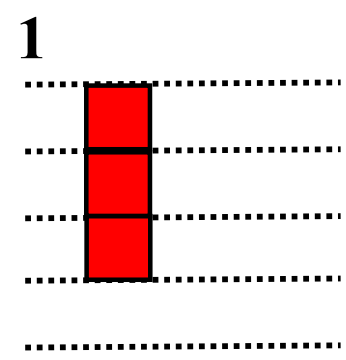

(a)

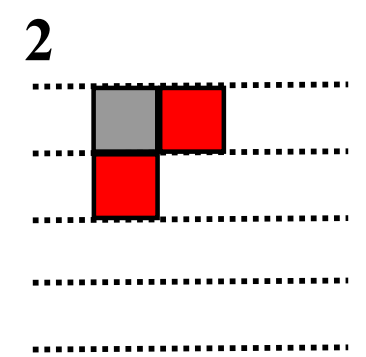

(b)

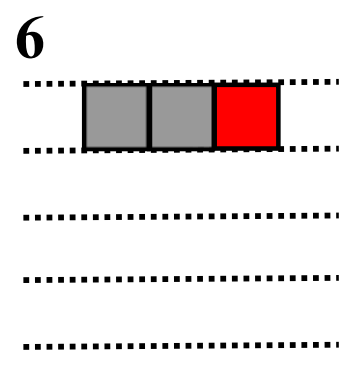

(c)

Figure 1. Tableaux for the coefficients appearing in this example. The red tiles in the tableaux are allowed to perform a permutation among the rows. The meaning of such permutations are to be discussed shortly.

where $\tilde{h}_{i}$ is obtained from $h_{i}$ with the replacement $\sigma_{4} \rightarrow \sigma_{4}^{\prime}$. From now on in this section we will simply write $\sigma_{4}$ for $\sigma_{4}^{\prime}$ for notational convenience.

The main idea of the method presented in [44] is to replace the contour integration

$$
\operatorname{Res}_{\left(\tilde{h}_{1}\right),\left(\tilde{h}_{2}\right),\left(\tilde{h}_{3}\right),\left(\sigma_{1}\right)}=\oint \frac{d \sigma_{1} \wedge \cdots \wedge d \sigma_{3} \wedge d \sigma_{4}^{\prime}}{\tilde{h}_{1} \cdots \tilde{h}_{3} \sigma_{1}}
$$

with a differential operator $\mathbb{D}$, which should be of the third-order in this particular case and can be written as

$$
\mathbb{D}=\sum_{\substack{0 \leq r_{i} \leq 3 \\ r_{1}+r_{2}+r_{3}+r_{4}=3}} a_{r_{1}, r_{2}, r_{3}, r_{4}}\left(\frac{\partial}{\partial \sigma_{1}}\right)^{r_{1}}\left(\frac{\partial}{\partial \sigma_{2}}\right)^{r_{2}}\left(\frac{\partial}{\partial \sigma_{3}}\right)^{r_{3}}\left(\frac{\partial}{\partial \sigma_{4}}\right)^{r_{4}},
$$

where the coefficients $a$ 's are to be fixed by the local duality theorem and the intersection number equation. One of the main points of the current paper is to provide an efficient method to determine those coefficients without solving the local duality and intersection number equations by brute force.

Now we briefly describe how the shortcut method works out in this particular example. There are 20 coefficients $a_{r_{1}, r_{2}, r_{3}, r_{4}}$ to be determined. Before turning to the equations from local duality and intersection number, we firstly classify the coefficients as follows. For each coefficient $a$ we define its rank to be $R\left(a_{r_{1}, r_{2}, r_{3}, r_{4}}\right)=r_{1} ! r_{2} ! r_{3} ! r_{4} !$. Furthermore, we associate a tableau with each $a$. All tableaux (up to subscript permutation) for our example are shown in figure 1 . The three tableaux in figure 1 depict $a_{0,1,1,1}, a_{0,0,1,2}$ and $a_{0}, 0,0,3$ and their corresponding ones with permuted subscripts respectiely.

With the definition of the rank of coefficients and the tableaux representation, solving the equations of the $a$ 's can be carried out in a very specific way that is much more efficient than treating it as a general equation solving problem. Recall that the local duality theorem yields,

$$
\mathbb{D}\left(\sigma_{i} \sigma_{j} \sigma_{1}\right)=\mathbb{D}\left(\sigma_{i} \sigma_{j} \tilde{h}_{1}\right)=\mathbb{D}\left(\sigma_{i} \tilde{h}_{2}\right)=\mathbb{D} \tilde{h}_{3}=0 \quad 0 \leq i, j \leq 3 .
$$

We will see that the equation $\mathbb{D}\left(\sigma_{i} \sigma_{j} \sigma_{1}\right)=0$ simply leads to the vanishing of all $a_{r_{1}, r_{2}, r_{3}, r_{4}}$ with $r_{1} \geq 1$. So this means, for a non-zero $a$, the number of tiles in the first row in 


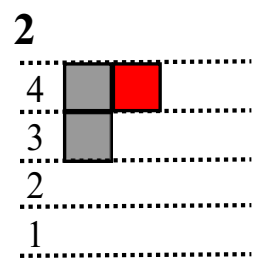

\section{2}

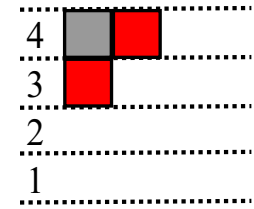

2

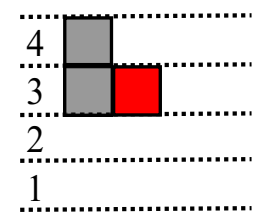

(a)

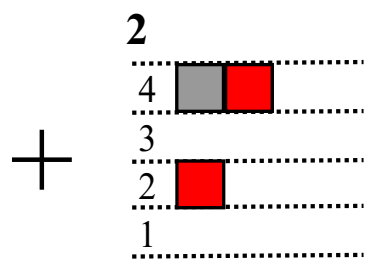

2

(b)

Figure 2. Tableaux indicating the permutations of the red tiles among the rows.

the corresponding tableau is zero. On the other hand, the equation $\mathbb{D} \tilde{h}_{3}=0$ means the vanishing of $a$ 's of rank 1 . Thus $a$ 's corresponding to the tableau in figure 1(a) are all vanishing. The equation $\mathbb{D}\left(\sigma_{i} \sigma_{i} \tilde{h}_{1}\right)=0$ will give us a relation between $a$ 's of rank 6 and $a$ 's of rank 2, e.g.

$$
a_{0,0,0,3}=\frac{-2 !}{3 ! \ell_{4}}\left(\ell_{1} a_{1,0,0,2}+\ell_{2} a_{0,1,0,2}+\ell_{3} a_{0,0,1,2}\right) .
$$

This equation can actually be read off directly from the tableau in figure 1(c). By permuting the red tiles of $a_{0,0,0,3}$ 's tableau among the rows, we could get the tableaux corresponding to $a_{1,0,0,2}, a_{0,1,0,2}$ and $a_{0,0,1,2}$ respectively. In terms of the coefficients, this exactly corresponds to (2.8). Namely, $a_{0,0,0,3}$ multiplied by its rank and $\ell_{4}$ equals the minus sum of $a_{1,0,0,2}$, $a_{0,1,0,2}$ and $a_{0,0,1,2}$ multiplied by their ranks and $\ell_{i}$, where $i$ indicates the row index of the red tile of the corresponding tableau.

The remaining unknown $a$ 's are those corresponding to the tableau figure 1(b) with rank 2. They are all the variables present in the intersection number equation

$$
\begin{aligned}
& 2 a_{0,0,1,2} \ell_{4} \ell_{3,4} \ell_{2,3,4}-2 a_{0,0,2,1} \ell_{3} \ell_{3,4} \ell_{2,3,4}+2 a_{0,2,0,1} \ell_{2} \ell_{2,4} \ell_{2,3,4} \\
- & 2 a_{0,1,0,2} \ell_{4} \ell_{2,4} \ell_{2,3,4}+2 a_{0,1,2,0} \ell_{3} \ell_{2,3} \ell_{2,3,4}-2 a_{0,2,1,0} \ell_{2} \ell_{2,3} \ell_{2,3,4}=6 .
\end{aligned}
$$

Other equations involving variables of rank two are $\mathbb{D}\left(\sigma_{i} \sigma_{j} \tilde{h}_{1}\right)=0$ with $i \neq j$ and $\mathbb{D}\left(\sigma_{i} \tilde{h}_{2}\right)=0$. For example $\mathbb{D}\left(\sigma_{3} \sigma_{4} \tilde{h}_{1}\right)=0$ is

$$
2 \ell_{4} a_{0,0,1,2}+2 \ell_{3} a_{0,0,2,1}=0
$$

where we have used the fact that $a_{0,1,1,1}$ and $a_{1,0,1,1}$ are zero. This equation is represented by figure $2(\mathrm{a})$ in the same way as that for eq. (2.8). This equation tells us that the first two terms in the eq. (2.9) are the same. The equation $\mathbb{D}\left(\sigma_{4} \tilde{h}_{2}\right)=0$ is, after ignoring the zero variables,

$$
2 \ell_{3,4} a_{0,0,1,2}+2 \ell_{2,3} a_{0,1,0,2}=0 .
$$


Similarly this equation can also be read off from the tableau. For that purpose, we paint red the two tiles as shown in figure 2(b) and permute the red tiles among the four rows. To get eq. (2.11), we simply take the sum of the products of the corresponding $a$ 's with their ranks and corresponding $\ell_{i, j}$ 's, where $i, j$ are the row indices of the red tiles of respective tableaux obtained through the tile moving described above. Accordingly, it is also seen that the first term and the forth term on the left hand side of eq. (2.9) are equal. By considering all the equations of $\mathbb{D}\left(\sigma_{i} \sigma_{j} \tilde{h}_{1}\right)=0$ with $i \neq j$ and $\mathbb{D}\left(\sigma_{i} \tilde{h}_{2}\right)=0$ in a similary way, we can see that all the terms in the intersection number equation are actually equal. Hence the solutions of those $a$ 's appearing in (2.9) are just the inverses of their respective coefficients. Up to this point, we thus have already solved all the $a$ 's and found the exact form of the operator $\mathbb{D}$. Now we can apply the $\mathbb{D}$ operator to evaluate the generalized CHY integrand,

$$
\begin{aligned}
\mathcal{I}_{4}^{1} & =\frac{-\langle 12\rangle^{4}}{\langle 12\rangle\langle 23\rangle\langle 34\rangle\langle 45\rangle\langle 51\rangle} \oint \frac{d \sigma_{1} \wedge \cdots \wedge d \sigma_{3} \wedge d \sigma_{4}}{\tilde{h}_{1} \cdots \tilde{h}_{3} \sigma_{1}} \frac{\sigma_{3}\left(\sigma_{2}-\sigma_{4}\right)}{\sigma_{4}-1} \\
& =\frac{-\langle 12\rangle^{4}}{\langle 12\rangle\langle 23\rangle\langle 34\rangle\langle 45\rangle\langle 51\rangle} 2 a_{0,0,1,2}=\frac{-\langle 12\rangle^{4}}{\langle 12\rangle\langle 23\rangle\langle 34\rangle\langle 45\rangle\langle 51\rangle} \ell_{4} \ell_{3,4} \ell_{2,3,4} .
\end{aligned}
$$

\subsection{Preliminary: notations \& tableaux}

In the previous discussion, the one-loop SYM integrand for four external particles is massaged and turned into a sum of several terms, all of which are of the following form,

$$
\mathcal{I}_{n}^{l=1}=\oint_{h_{1}=\cdots h_{n-1}=h_{n}^{\prime}=0} \frac{d \sigma_{1} \wedge \cdots \wedge d \sigma_{n-1} \wedge d \sigma_{n}^{\prime}}{h_{1} \cdots h_{n-1} h_{n}^{\prime}} \mathcal{H}\left(\sigma_{1}, \ldots, \sigma_{n-1}, \sigma_{n}^{\prime}\right),
$$

where $\sigma_{j}(j=1, \cdots, n-1)$ are the $(n-1)$ variables that need to be integrated out (with the gauge choice $\sigma_{n}=1$ ) and $\sigma_{n}^{\prime}$ is the auxiliary parameter that comes into play in the homogenization of the scattering equations (for details, see section 4.2 and 4.3 of [44]). $h_{j}$ $(j=1, \cdots, n-1)$ denote the $(n-1)$ polynomial scattering equations (3.6) that are necessary to capture the behavior of an $n$-point scattering and $h_{n}^{\prime}=\sigma_{i}(i=1, \cdots, n-1)$ or $h_{n}^{\prime}=\sigma_{n}^{\prime}$. In this form, the divisors are generated either by one of the polynomial scattering equations or by the simplest monomial possible. As reviewed in the introduction, such contour integrations are associated with a differential operator with a certain number of coefficients fixed by the local duality theorem and the intersection number. These constraints are all linear and especially the local duality theorem gives rise a sparse constraint matrix, which in general can be solved by various sparse matrix methods. In the case of an integral in the form of (2.13), thanks to the beautiful mathematical structures of scattering equations, these constraints can indeed be solved analytically. In this section we present the method that reduces considerably the size of the constraint matrix and obtains quickly the analytic solutions of the coefficients. For this reason, we would like to call an expression of form (2.13) prepared. Moreover, as we will observe in more examples, the CHY-like representations for amplitudes/integrands can often be reduced to the prepared form or similar ones with a slightly modified $h_{n}^{\prime}$, even though the original expressions seem much more complicated. For an expression with a modified $h_{n}^{\prime}$ which still remains a monomial, we expect our algorithm can be generalized. 
288

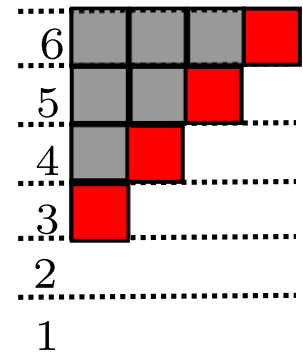

(a)
288

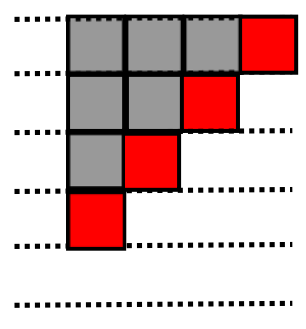

(b)

Figure 3. Box diagram.

As shown in the discussion of the four-point one-loop integrand, to demonstrate our method in a more intuitive way, a graphical representation of the coefficients in the operator comes in handy. Let us take a coefficient $a_{0,1,2,3,4}$ as an example and address a few details of its corresponding tableau depicted in figure $3(\mathrm{a})$. The $i$-th row of the tableau corresponds to the $i$-th index of the coefficient while the number of the tiles in the $i$-th row is equal to the value of the $i$-th index. The total number of tiles is the order of the corresponding differential operator. As mentioned above, we define the rank of coefficient $a_{r_{1}, r_{2}, \cdots, r_{n}}$ as the following integer number,

$$
R\left(a_{r_{1}, r_{2}, \cdots, r_{n}}\right)=r_{1} ! r_{2} ! \cdots r_{n} !
$$

and this number is written on top of the corresponding tableau. Sometimes we drop the row labels in the tableau as shown in figure 3(b), and this tableau represents the class of $a$ 's whose indices are related by permutations. For instance, the tableau in figure $3(\mathrm{~b})$ is corresponding to the coefficients $\left\{a_{\rho(0,0,1,2,3)}\right\}_{\rho \in S_{5}}$ where $S_{5}$ is the permutation group of five items and $\rho(0,0,1,2,3)$ means a permuation of the five digits $(0,0,1,2,3)$.

As shown in figure 3 , we have painted some tiles red in the tableaux. For a given unpainted tableau, we associate with it a class of colored tableaux which are obtained in the following way: in each row only the rightmost tile can be painted red, and we can choose to paint it or not. So altogether we have $2^{J}$ colored tableaux for each given unpainted one, where $J$ is the number of nonempty rows in the unpainted tableau. From each colored tableau, we can get several new tableaux (without considering their coloring) by permuting the red tiles among the rows. For example, in figure 4 are depicted the new tableaux resulted from the tile moving of the first colored tableau. In this example, we can obtain $a_{0,0,1,2,3}, a_{0,0,2,1,3}, a_{0,1,1,1,3}$ and $a_{1,0,1,1,3}$ from $a_{0,0,1,1,4}$.

So far we have played with the tableaux. Now we demonstrate how the tile moving can acquire actual meanings from the local duality theorem. Recall that the polynomial scattering equation of degree $m$ for $n$ particles takes the following form (throughout this paper we adopt the gauge choice $\sigma_{n}=1$ and all the polynomial scattering equations, if not specified otherwise, are homogenized with an auxiliary parameter $\sigma_{n}^{\prime}$; for notational 


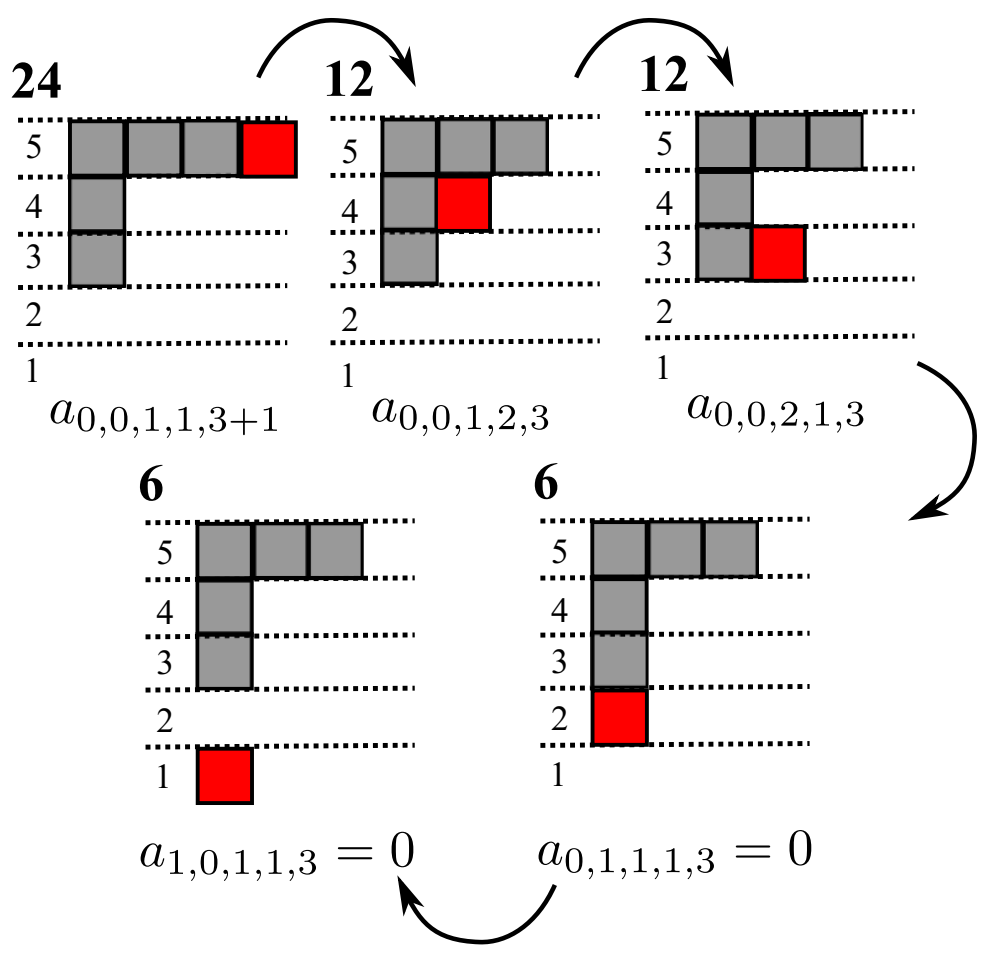

Figure 4. Example of a tile move.

compactness, we drop the prime and simply denote the auxiliary as $\sigma_{n}$ from now on),

$$
h_{m}=\sum_{1 \leqslant i_{1}<i_{2}<\cdots<i_{m} \leqslant n} \ell_{i_{1} i_{2} \cdots i_{m}} \sigma_{i_{1}} \sigma_{i_{2}} \cdots \sigma_{i_{m}} .
$$

The order of the differential operator $\mathbb{D}$ associated with the prepared form for $n$ points is

$$
M=1+2+\cdots(n-2)=\frac{(n-1)(n-2)}{2} .
$$

Since the differential operator $\mathbb{D}$ acting on a monomial in $\sigma_{i}$ 's does nothing but picking out the exponents of $\sigma_{i}$ 's, the local duality theorem yields,

$$
\sum_{i_{1}<i_{2}<\cdots i_{m}} \ell_{i_{1} i_{2} \cdots i_{m}} \underbrace{\left(r_{1} ! \cdots\left(r_{i_{1}}+1\right) ! \cdots\left(r_{i_{m}}+1\right) ! \cdots r_{n} !\right)}_{R\left(a_{r_{1}, \cdots,\left(r_{i_{1}}+1\right), \cdots,\left(r_{i_{m}}+1\right), \cdots, r_{n}}\right)} a_{r_{1}, \cdots,\left(r_{i_{1}}+1\right), \cdots,\left(r_{i_{m}}+1\right), \cdots, r_{n}}^{r_{1}}=0,
$$

where $r_{j}$ 's are non-negative integers satisfying the Frobenius equation $r_{1}+r_{2}+\cdots r_{n}=$ $M-m$. Here we see that the rank of $a_{r_{1}, \cdots, r_{n}}$ is merely the products of the factorials that arises when taking derivatives multiple times. There are $C_{n}^{m}$ coefficients $a_{r_{1}, \cdots, r_{n}}$ 's on the right-hand side above and their corresponding tableaux all have $r_{j}$ black tiles in the row $j$ and $m$ red tiles that sit in the $m$ different rows $\left\{i_{1}, i_{2}, \cdots, i_{m}\right\}$. The tableaux corresponding to these $a$ 's are exactly those related by permuting the red tiles as mentioned 
above! In other words, given a tableau that has $r_{j}$ black tiles in the $j$-th row and the rows $\left\{i_{1}, \cdots, i_{m}\right\}$ have one red tile each, there is a unique equation following from the local duality theorem that relates this tableau and those obtained by permuting the red tiles. Furthermore, scanning over the monomials of the correct degrees to construct the local duality equations is equivalent to exhausting all the legitimate colorings and writing down the corresponding equations.

As mentioned above, the equations arising from the local duality theorem are not independent from each other and the number of equations is much larger than the number of coefficients. With the correspondence between the colorings and the local duality constraints established, we are ready to present a method that systematically selects just enough independent local duality equations to fix the coefficients completely.

\subsection{Coefficient generating algorithm}

In this section, we spell out our method that analytically solves the local duality and the intersection number constraints associated with a prepared form and display the general solutions of the coefficients in the corresponding differential operator.

Although there are several possible colored tableaux associated with a given uncolored tableau, there is a preferred one that leads (by shuffling the red tiles among the rows) to new tableaux whose ranks are either lower than or equal to that of the original. This particular colored tableau is constructed as follows. For convenience let us assume $a_{r_{1}, r_{2}, \cdots, r_{n}}$ is a coefficient with indices such that $r_{i_{1}} \leqslant r_{i_{2}} \leqslant \cdots \leqslant r_{i_{n}}$. In the prefered colored tableau, we paint red the last tile in row $i_{n}$. Now for the other rows, if the row $i_{p}$ has one red tile and $r_{i_{p}}-r_{i_{p-1}} \leqslant 1, r_{i_{p-1}}>0$, we should also paint red the last tile of the row $r_{i_{p-1}}$; otherwise all the tiles in the row $r_{i_{p-1}}$ remain black and the coloring process stops here.

There are two types of the coefficients $a_{r_{1}, r_{2}, \cdots, r_{n}}:$ let $\left(j_{1}, j_{2}, \cdots j_{n}\right)$ be a particular permutation of the indices such that $r_{j_{1}} \leqslant r_{j_{2}} \leqslant \cdots \leqslant r_{j_{n}}$; if $r_{j+1}-r_{j} \leqslant 1$ for $j=1, \cdots n-1$, $a_{r_{1} r_{2} \cdots r_{n}}$ is called elementary; otherwise it is called non-elementary. It is easy to observe that, for an elementary coefficient, the aforementioned preferred coloring relates it with those of lower or equal ranks. A non-elementary coefficient gets turned into only lowerrank ones through the preferred coloring. Moreover, for a particular prepared form for $n$ points, the corresponding tableaux are all of $M=(n-1)(n-2) / 2$ tiles in total. Up to row permutation, there is a unique tableau with the highest rank among the elementary ones, that is the tableau associated with $a_{0,0,1,2,3, \ldots, n-2}$ whose rank is $R_{e}=(n-2) !(n-1) ! \cdots 1$ !.

The above observations about the prepared form actually provide us a natural solving order in which the local duality constraints can be conveniently solved. In the following discussion, we will show that in the operator associated with a prepared form, any coefficients with ranks lower than $R_{e}$ must vanish while the elementary ones with rank $R_{e}$ can be easily worked out, which leaves us only non-elementary ones with ranks greater than $R_{e}$. Those higher rank coefficients can however be obtained by an inductive method: suppose we have obtained all the coefficients - both elementary and non-elementary — up to some rank $R_{0} \geqslant R_{e}$ and the next possible rank is $R_{1}$, then a non-elementary coefficient of rank $R_{1}$ whose tableau is painted in the preferred way can be directly read off from the relation associated with the colored tableau, i.e. it can be represented in terms of other coefficients 
of lower ranks only. This procedure continues all the way up to the highest-rank coefficients and thus this way we can determine all the coefficients analytically one after another.

Trivial coefficients. In general, the differential operator $\mathbb{D}$ may be very complicated. However, it is easy to see that many coefficients in $\mathbb{D}$ are actually zero, i.e. trivial, when $\mathbb{D}$ is associated with a form that is prepared (see (2.13)).

Firstly, for a prepared form with $h_{n}=\sigma_{i}$, the coefficients $a_{r_{1}, \cdots, r_{n}}$ with $r_{i} \geqslant 1$ must vanish, because the local duality theorem for $h_{n}$ yields,

$$
\mathbb{D}\left(\sigma_{1}^{r_{1}} \cdots \sigma_{n}^{r_{n}} h_{n}\right)=a_{r_{1}, r_{2}, \cdots, r_{i-1}, r_{i}+1, r_{i+1}, \cdots, r_{n}}=0
$$

where $r_{j}$ are non-negative solutions to $r_{1}+\cdots+r_{n}=M-1$.

Secondly, the coefficients $a_{r_{1}, \cdots, r_{n}}$ must vanish if $R\left(a_{r_{1}, \cdots, r_{n}}\right)<R_{e}$. To see this, let us consider a prepared form with $h_{n}=\sigma_{i}$. Since $r_{1}+\cdots+r_{n}=M$, the lowest-rank coefficients must be elementary and have $r_{i} \geqslant 1$ when $n \geqslant 5$ (when $n=4$ the lowest-rank coefficient has the $i$-th index being 0 and all the rest 1 ), which obviously vanish. Now we only need to show that every elementary coefficient of the form $a_{r_{1}, \cdots, r_{i-1}, 0, r_{i+1} \cdots r_{n}}$ (where $r_{1} ! \cdots r_{n}$ ! $<R_{e}$ ) is zero, since the non-elementary ones can be written in terms of coefficients with lower ranks only. Let $\left\{i_{1}, \cdots, i_{i-1}, i_{i+1}, \cdots, i_{n}\right\}$ be a permutation of $\{1, \cdots, i-1, i+1, \cdots, n\}$ such that $r_{i_{1}} \leqslant \cdots \leqslant r_{i_{n}}$ and $r_{i_{j+1}}-r_{i_{j}} \leqslant 1, r_{i_{i+1}}-r_{i_{i-1}} \leqslant 1$. Then we must have $r_{i_{n}} \leqslant n-3$ in order to guarantee that the rank is lower than $R_{e} \cdot{ }^{1}$ On the other hand, we must also have $r_{i_{1}}=1$ because $r_{2}+\cdots+r_{n}=M=\operatorname{ord}(\mathbb{D}) .^{2}$ Hence the tableau for $a_{r_{1}, \cdots, r_{i-1}, 0, r_{i+1}, \cdots, r_{n}}$ has only the $i$-th row empty and all the other rows filled. The aforementioned preferred coloring leads to $(n-1)$ red tiles in total and the local duality relation represented by this colored tableau involves $a_{r_{1}, \cdots, r_{i_{1}}, 0, r_{i+1}, \cdots, r_{n}}$ and other coefficients that all have $r_{i}=1$, i.e. $a_{r_{1}, \cdots, r_{i-1}, 0, r_{i+1}, \cdots, r_{n}}$ is rewritten as a linear combination of several vanishing $a$ 's and therefore is zero. By induction, all the non-elementary coefficients with ranks lower than $R_{e}$ are also zero.

Non-trivial elementary coefficients. Now we turn to the elementary coefficients of rank $R_{e}$. Recall that the intersection number constraint reads

$$
\mathbb{D}\left[\operatorname{det}\left(\partial_{i} h_{j}\right)\right]=(n-1) !,
$$

where $h_{n}=\sigma_{i}$ and the scattering equations $h_{j}(j=1, \cdots n-1)$ are polynomials in $\sigma_{i}$ 's of degree $j$ respectively. Using the explicit forms of $h$ 's, we can see that only coefficients of ranks no greater than $R_{e}$ appear in (2.18). Combining with the previous discussion about

${ }^{1}$ If $r_{i_{n}}=n-2$ and all the other $r_{j}$ 's saturate their lower bounds, we have

$$
R\left(a_{0, r_{2} \cdots r_{n}}\right)=r_{i_{n}} ! r_{i_{n-1}} ! \cdots r_{i_{1}} !=(n-2) !(n-3) ! \cdots 1 ! .
$$

${ }^{2}$ Likewise, if $r_{i}=0$ and all the other $r_{j}$ 's saturate their upper bounds, we have

$$
r_{1}+\cdots+r_{n}=r_{i_{2}}+\cdots r_{i_{n}}=0+1+\cdots+(n-3)+(n-3)<\frac{(n-1)(n-2)}{2}=M .
$$

Therefore we must have $r_{i_{1}}=1$ and $r_{i_{n}} \leqslant n-3$. 


\section{$(n-2) !(n-3) ! \ldots 1 ! 0 !$}

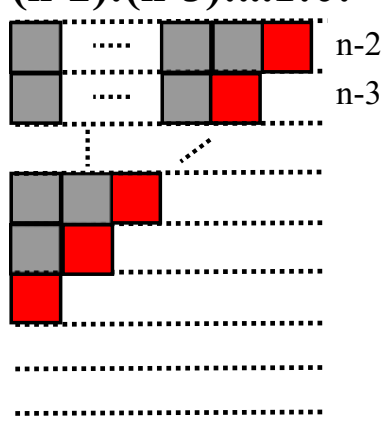

Figure 5. The tableaux $a_{\{0,0,1,2, \cdots, n-3, n-2\}}$.

trivial coefficients, we know that the only surviving coefficients in the intersection number equation are of the form

$$
a_{\rho(0), \rho(1), \ldots, \rho(i-2), 0, \rho(i-1), \ldots, \rho(n-2)}
$$

where $\rho$ is a permutation of $\{0,1,2, \ldots, n-2\}$, i.e. all $a$ 's with the $i$-th index zero and other indices a permutation of $\{0,1,2, \ldots, n-2\}$. These are elementary and their corresponding tableaux are shown in figure 5 .

The intersection number equation (2.18) is then

$$
\begin{aligned}
(n-2) ! \cdots 2 ! 1 ! 0 ! \sum_{\rho}(-1)^{\operatorname{sgn}(\rho)} \ell_{v_{n-2}^{(\rho)}} \ell_{v_{n-3, n-2}^{(\rho)}} \cdots \ell_{v_{1,2, \cdots, n-3, n-2}^{(\rho)}} \ell_{1, \cdots, i-1, i, \cdots, n} & \\
& \times a_{\rho(0), \rho(1), \ldots, \rho(i-2), 0, \rho(i-1), \ldots, \rho(n-2)}=(n-1) !
\end{aligned}
$$

where $v_{j_{1}, \cdots, j_{r}}^{(\rho)}$ is the position set of the number set $\left\{j_{1}, \cdots, j_{r}\right\}$ among the indices of $a_{\rho(0), \ldots, \rho(i-2), 0, \rho(i-1), \ldots, \rho(n-2)}$. A pair of such $a_{\rho(0), \rho(1), \ldots, \rho(i-2), 0, \rho(i-1), \ldots, \rho(n-2)}$ 's are directly related by the local duality theorem, as long as their indices are related by permutations. To see this, let us consider the following two permutations,

$$
\begin{aligned}
& \rho_{1}\{0,1,2,3 \cdots, n-2\}=\{0,1, \cdots, n-3, \cdots, n-2\}, \\
& \rho_{2}\{0,1,2,3 \cdots, n-2\}=\{0,1, \cdots, n-2, \cdots, n-3\} .
\end{aligned}
$$

The right-hand sides of the two equations above are associated with each other by swapping $(n-3)$ and $(n-2)$ and the local duality theorem associated with $h_{1}$ yields,

$$
\mathbb{D}\left(\sigma_{1}^{m_{1}} \cdots \sigma_{i-1}^{m_{i-1}} \sigma_{i+1}^{m_{i+1}} \cdots \sigma_{n}^{m_{n}} h_{1}\right)=0,
$$

where $m_{j}=\min \left(\rho_{1}(j), \rho_{2}(j)\right),(j=0,1,2, \cdots, n-2)$ and in particular $m_{v_{n-2}^{\left(\rho_{1}\right)}}=(n-3)$, $m_{v_{n-2}^{\left(\rho_{2}\right)}}=(n-3)$. Only two terms survive on the left-hand side of $(2.23)$ and this gives the pairwise relation among the non-trivial elementary coefficients as follows,

$\operatorname{sgn}\left(\rho_{1}\right) \ell_{v_{n-2}^{\left(\rho_{1}\right)}} a_{\rho_{1}(0), \ldots, \rho_{1}(i-2), 0, \rho_{1}(i-1), \ldots, \rho_{1}(n-2)}=\operatorname{sgn}\left(\rho_{2}\right) \ell_{v_{n-2}^{\left(\rho_{2}\right)}} a_{\rho_{2}(0), \ldots, \rho_{2}(i-2), 0, \rho_{2}(i-1), \ldots, \rho_{2}(n-2)} \cdot$ 
Now we consider another two permutations,

$$
\begin{aligned}
& \rho_{1}\{0,1,2,3 \cdots, n-2\}=\{\cdots, n-4, \cdots, n-3, \cdots, n-2\}, \\
& \rho_{2}\{0,1,2,3 \cdots, n-2\}=\{\cdots, n-3, \cdots, n-2, \cdots, n-4\},
\end{aligned}
$$

where the right-hand sides are related by two permutation actions and the corresponding equation is given by the local duality theorem for $h_{2}$ which reads,

$$
\mathbb{D}\left(\sigma_{1}^{m_{1}} \cdots \sigma_{i-1}^{m_{i-1}} \sigma_{i+1}^{m_{i+1}} \cdots \sigma_{n}^{m_{n}} h_{2}\right)=0,
$$

where $m_{j}$ is defined the same way as above and for this case we have $m_{v_{n-3}^{\left(\rho_{2}\right)}}=(n-4)$, $m_{v_{n-2}^{\left(\rho_{2}\right)}}=(n-3)$ and $m_{v_{n-2}^{\left(\rho_{1}\right)}}=(n-4)$. This equation also results in a relation for a pair of non-trivial elementary coefficients as follows,

$$
\begin{aligned}
& \operatorname{sgn}\left(\rho_{1}\right) \ell_{v_{n-3, n-2}^{\left(\rho_{1}\right)}} a_{\rho_{1}(0), \ldots, \rho_{1}(i-2), 0, \rho_{1}(i-1), \ldots, \rho_{1}(n-2)}= \\
& \quad \operatorname{sgn}\left(\rho_{2}\right) \ell_{v_{n-3, n-2}^{\left(\rho_{2}\right)}} a_{\rho_{2}(0), \ldots, \rho_{2}(i-2), 0, \rho_{2}(i-1), \ldots, \rho_{2}(n-2)} .
\end{aligned}
$$

By induction, we can construct the relations between any given pair of non-trivial elementary coefficients, since they all have the same indices up to permutations. Now it is straightforward to eliminate all but one coefficient in the intersection number equation and solve this constraint right away, which gives rise to the general solution of the non-trivial elementary coefficients as follows,

$$
a_{\rho(0), \ldots, \rho(i-2), 0, \rho(i-1), \ldots, \rho(n-2)}=\frac{(-1)^{\operatorname{sgn}(\rho)}}{(n-2) ! \cdots 1 \ell_{v_{n-2}^{(\rho)}} \ell_{v_{n-3, n-2}^{(\rho)} \cdots} \cdots \ell_{v_{1,2, \cdots, n-3, n-2}^{(\rho)}} \ell_{1, \cdots, i-1, i+1 \cdots, n}} .
$$

Summary of the coefficient generating method. Now let us summarize our method that computes the coefficients $a_{r_{1}, \cdots, r_{n}}$ in the differential operator $\mathbb{D}$ for a prepared CHYtype expression for $n$ points with $h_{n}=\sigma_{i}$.

In figure 6 we show the flowchart indicating the order in which the coefficients are solved. As discussed above, among the non-vanishing coefficients, the lowest-rank ones are elementary whose tableaux are depicted as the leftmost one in figure 6 and their analytic solutions are given in (2.29). These elementary coefficients are our building blocks and from their corresponding tableaux we move towards the right end of the flowchart. The tableaux for the remaining coefficients are all painted in the preferred way and thus the solutions of these non-elementary coefficients following from the corresponding relation read (supposing that there are $k$ red tiles that sit in the rows $\left\{i_{1}, \cdots, i_{k}\right\}$ while the the number of the unpainted tiles in each row is $r_{i}$ ),

$$
\begin{aligned}
& a_{r_{1}, \cdots, r_{\left(i_{1}-1\right)},\left(r_{i_{1}}+1\right), r_{\left(i_{1}+1\right)}, \cdots r_{\left(i_{k}+1\right)},\left(r_{i_{k}}+1\right), r_{\left(i_{k}+1\right)}, \cdots, r_{n}}= \\
& \quad \frac{-1}{\ell_{i_{1} \cdots i_{k}} R\left(a_{\left.r_{1}, \cdots, r_{\left(i_{1}-1\right)},\left(r_{i_{1}}+1\right), r_{\left(i_{1}+1\right)}, \cdots r_{\left(i_{k}+1\right)},\left(r_{i_{k}}+1\right), r_{\left(i_{k}+1\right)}, \cdots, r_{n}\right)}\right)} \\
& \quad \times\left[\sum_{j_{1}<\cdots<j_{k},\{j\} \neq\{i\}} \ell_{j_{1} \cdots j_{k}} R(a) a_{r_{1}, \cdots, r_{\left(j_{1}-1\right)},\left(r_{j_{1}}+1\right), r_{\left(j_{1}+1\right)}, \cdots r_{\left(j_{k}+1\right)},\left(r_{j_{k}}+1\right), r_{\left(j_{k}+1\right)}, \cdots, r_{n}}\right],
\end{aligned}
$$




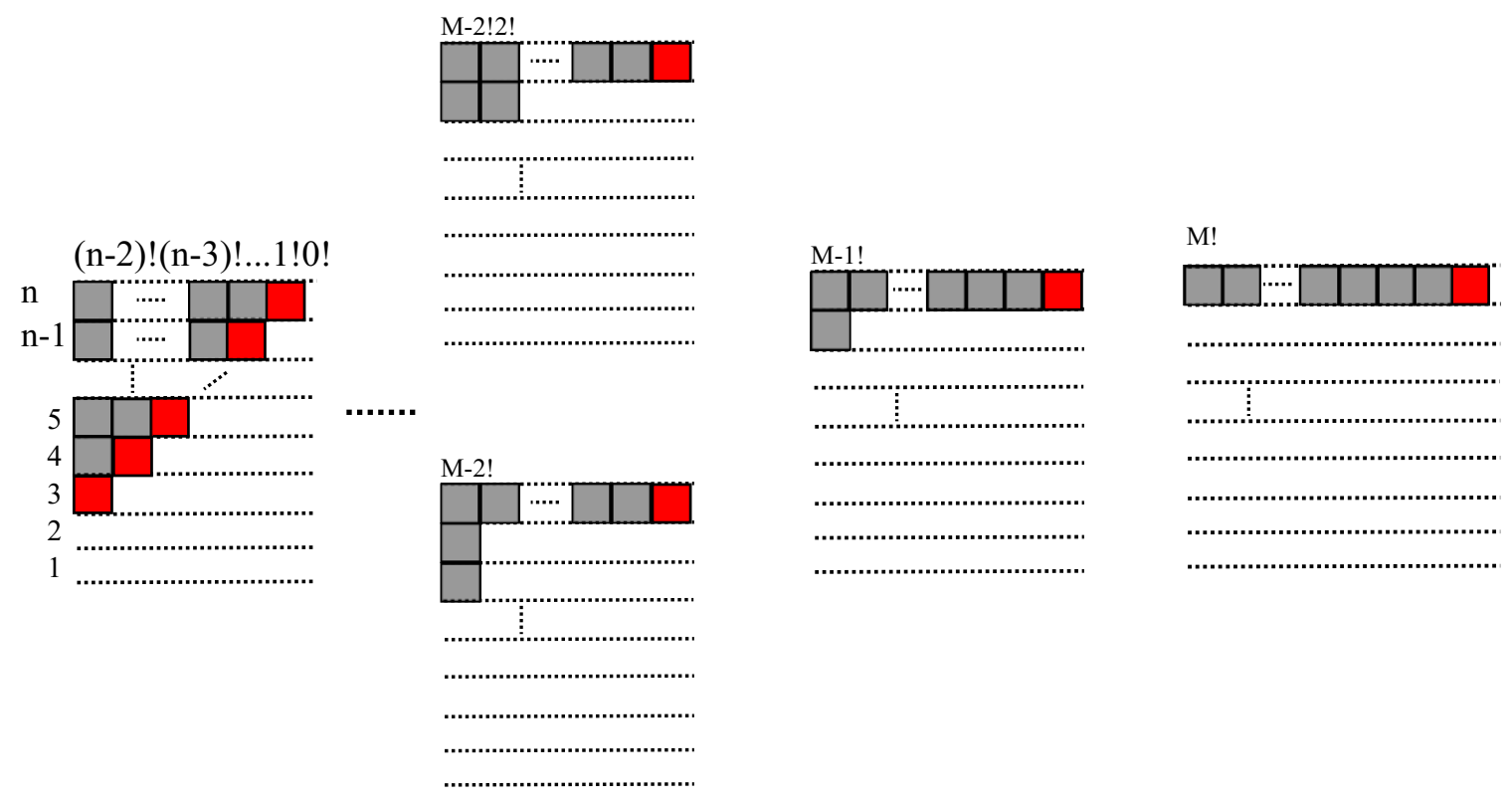

Figure 6. The tableaux of the non-zero $a$ 's for the prepared form in $n$-point amplitude.

where $R(a)$ denotes the rank of the coefficient it multiplies. According to the previous

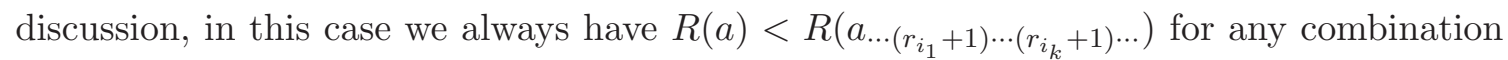
of $\left\{j_{1}, \cdots, j_{k}\right\}$ since $a_{\cdots}\left(r_{i_{1}}+1\right) \cdots\left(r_{i_{k}}+1\right) \cdots$ is non-elementary.

The relations corresponding to the painted tableaux in figure 6 are independent from each other, since every relation involves a new coefficient. These relations are obviously complete as well, since they are linear and fix all the coefficients uniquely.

\section{One-loop five-point SYM amplitude}

In this and the next sections, we further illustrate the aforementioned method by discussing more examples, the one-loop MHV amplitude for five external particles in $\mathcal{N}=4 \mathrm{SYM}$ and the $n$-gon amplitude for an arbitrary number of particles. At first sight, the one-loop SYM integrand [14] might appear considerably more complicated than the four-point case due to the existence of the Pfaffian. Fortunately for us, supersymmetries, which enter the story in the GSO projection, simplify the integrand/amplitude by a great deal and cast the integrand into a form that is no longer intimidating at all. We first demonstrate this process using the relations among the Jacobi theta functions studied in [50] and the decomposition of the polarization vectors. In the end we arrive at an expression that is a sum of prepared forms and the evaluation of this expression becomes straightforward.

\subsection{Simplify the one-loop generalized CHY integrand}

The generalized CHY form for the one-loop 5-point integrand in SYM is first conjectured as the following by Geyer et al. in $[14,15]$ and checked against the corresponding Q-cut 
expressions up to five points,

$$
\mathcal{I}_{5}^{l=1}=\oint \frac{d \sigma_{1} \cdots d \sigma_{4}}{f_{1} \cdots f_{4}} \mathcal{F} \mathrm{PT}_{5} \prod_{i=1}^{4} \frac{1}{\sigma_{i}}
$$

where $\mathrm{PT}_{5}$ is the well-known Parke-Taylor factor for five points and its general expression reads

$$
\mathrm{PT}_{n}=\sum_{\rho \in \mathcal{S}_{n}} \frac{1}{\sigma_{\rho(1)}\left(\sigma_{\rho(1)}-\sigma_{\rho(2)}\right)\left(\sigma_{\rho(2)}-\sigma_{\rho(3)}\right) \cdots\left(\sigma_{\rho(n-1)}-\sigma_{\rho(n)}\right)},
$$

in which $\mathcal{S}_{n}$ is the cyclic permutation group of $n$ elements. The shorthand notation $\mathcal{F}$ denotes the summation of the Pfaffians over the GSO sectors that have even spin structures, namely $\mathcal{F}=\left.\sum_{\alpha} c_{\alpha} \mathcal{Z}_{\alpha} \operatorname{Pf}\left(W_{\alpha}\right)\right|_{\tau \rightarrow \infty}$ where $\tau$ is regarded simply as a parameter in the SYM computations, but can be interpreted as the moduli parameter of the corresponding oneloop worldsheet in string theory. ${ }^{3} \mathcal{Z}_{\alpha}$ denotes the theory-specific partition functions for the GSO sectors and in the case of $\mathcal{N}=4$ super Yang-Mills reads

$$
\mathcal{Z}_{\alpha}=\frac{\theta_{\alpha}(0 \mid \tau)^{4}}{\eta(\tau)^{12}}, \quad \alpha=2,3,4
$$

where $\eta(\tau)$ is the Dedekind eta function and $\alpha$ labels the GSO sectors with even spin structures, that is, $(R+),(N S+),(N S-)$ respectively. The coefficients $c_{\alpha}$ take care of the GSO projection, and thus take the values $\left\{c_{2}, c_{3}, c_{4}\right\}=\{+1,-1,+1\}$. The matrix in the Pfaffian takes the following skew-symmetric form,

$$
W_{\alpha}=\left(\begin{array}{cc}
A & -C^{T} \\
C & B
\end{array}\right)
$$

where

$$
A_{i j}=\left\{\begin{array}{l}
\epsilon_{i} \cdot \epsilon_{j} \mathcal{S}_{\alpha}\left(z_{i j} \mid \tau\right) \\
0
\end{array}, \quad B_{i j}=\left\{\begin{array}{l}
k_{i} \cdot k_{j} \mathcal{S}_{\alpha}\left(z_{i j} \mid \tau\right) \\
0
\end{array}, \quad C_{i j}=\left\{\begin{array}{ll}
\epsilon_{i} \cdot k_{j} \mathcal{S}_{\alpha}\left(z_{i j} \mid \tau\right) & i \neq j \\
-\epsilon_{i} \cdot F_{i}\left(z_{i}\right) & i=j
\end{array},\right.\right.\right.
$$

where the polarization vectors and the momenta of the external gluons are denoted as $\epsilon$ 's and $k$ 's (without loss of generality, we choose the helicities of $\epsilon_{1}$ and $\epsilon_{2}$ to be negative and the rest positive throughout this section). $\mathcal{S}_{\alpha}$ denotes the fermionic propagator in the RNS formalism of string theory, with $\alpha$ labeling the sector, whose explict form will not be used in this paper. We have also re-parameterized the variables as $\sigma=e^{2 \pi i\left(z-\frac{\tau}{2}\right)}$ and the scattering equations in terms of the complex variables $z_{i}$ read

$$
\left.f_{i} \equiv \frac{k_{i} \cdot F_{i}}{\sigma_{i}}\right|_{\tau \rightarrow \infty}, \quad k_{i} \cdot F_{i}\left(z_{i}\right)=k_{i} \cdot \ell+\frac{1}{2 \pi i} \sum_{j \neq i} k_{i} \cdot k_{j} \mathcal{R}_{i j}
$$

where $\mathcal{R}_{i j} \equiv-\partial_{z_{i}} \mathcal{G}\left(z_{i j} \mid \tau\right)=\frac{\theta_{1}^{\prime}\left(z_{i j} \mid \tau\right)}{\theta_{1}\left(z_{i j} \mid \tau\right)}$, with $\mathcal{G}$ denoting the bosonic propagator.

\footnotetext{
${ }^{3}$ This limit is equivalent to the $q \rightarrow 0$ limit in [14].
} 
Now we begin to simplify the Pfaffian (3.4), which by definition is a polynomial in the elements of the matrix (3.4). The terms in this polynomial are all of the following form with coefficients containing the kinetic informations only,

$$
c(\{\epsilon\},\{k\}) \mathcal{S}_{i_{1} i_{1}^{\prime} i_{2} i_{2}^{\prime} \cdots i_{m} i_{m}^{\prime}}^{\alpha} C_{j_{1} j_{1}} \cdots C_{j_{5-m} j_{5-m}},
$$

where $\mathcal{S}_{i_{1} i_{1}^{\prime} i_{2} i_{2}^{\prime} \cdots i_{m} i_{m}^{\prime}}^{\alpha}=\mathcal{S}_{\alpha}\left(z_{i_{1} i_{1}^{\prime}} \mid \tau\right) \cdots \mathcal{S}_{\alpha}\left(z_{i_{m} i_{m}^{\prime}} \mid \tau\right)$ and $\left(i_{1}^{\prime}, \cdots, i_{m}^{\prime}\right)$ is a permutation of $\left(i_{1}, \cdots, i_{m}\right)$. The diagonal terms $C_{l l}$ of the off-diagonal blocks in (3.4) are the only ones that contribute factors other than $\mathcal{S}_{\alpha}$. The union of all the indices above is simply $\{1,2,3,4,5\}$ with each number appearing twice while the sets of indices $\left\{i_{1}, \cdots, i_{m}\right\}$ and $\left\{j_{1}, \cdots j_{5-m}\right\}$ have no overlap. The coefficients $c(\{\epsilon\},\{k\})$ and the entries $C_{l l}$ are evidently universal for all GSO sectors and can be pulled out from the the summation over the spin structures; the only factors relevant for the GSO summation are the products of $\mathcal{S}_{\alpha}$ 's. Such summations are worked out explicitly at one loop for the $\mathcal{N}=4$ partition functions in [50], exploiting the properties of the Jacobi theta functions. For $m \leq 3$ the weighted sum simply vanishes and thus the surviving terms in the five-point Pfaffian are those with $m=4$ or $m=5$, which sum up to the following simple results when $z_{i_{1} i_{1}^{\prime}}+\cdots z_{i_{m} i_{m}^{\prime}}=0$ (this condition is satisfied trivially in our case),

$$
\begin{aligned}
& \sum_{\alpha} c_{\alpha} \mathcal{Z}_{\alpha} S_{i_{1} i_{1}^{\prime} \cdots i_{4} i_{4}^{\prime}}^{\alpha}=(2 \pi)^{4} \\
& \sum_{\alpha} c_{\alpha} \mathcal{Z}_{\alpha} S_{i_{1} i_{1}^{\prime} \cdots i_{5} i_{5}^{\prime}}^{\alpha}=(2 \pi)^{4}\left(\mathcal{R}_{i_{1} i_{1}^{\prime}}+\cdots+\mathcal{R}_{i_{5} i_{5}^{\prime}}\right) .
\end{aligned}
$$

Using these relations, the sum of the Pfaffians corresponding to the MHV amplitude over the even spin structures can be directly written down as the following,

$$
\mathcal{F}=\sum_{\rho\{345\}}\langle 12\rangle^{2}[\rho(3) \rho(4)]^{2} \epsilon_{\rho(5)} \cdot \ell+\sum_{\rho\{2345\}} \frac{\langle 12\rangle^{4}[\rho(2) \rho(3)][\rho(4) \rho(5)]^{2}}{2 \pi i\langle 1 \rho(2)\rangle\langle 1 \rho(3)\rangle} \mathcal{R}_{\rho_{b}(2) \rho_{b}(3)},
$$

where $\rho\{345\}$ and $\rho\{2345\}$ are the permutation groups $S_{3} / S_{2}{ }^{4}$ and $S_{4} / S_{2} \times S_{2}$ respectively. This expression does not yet put all the external particles on the same footing. In the $\mathcal{N}=4$ SYM case, it is possible to make the cyclic symmetry manifest at the level of integrand, by projecting the polarization vectors $\epsilon_{i}^{\mu}$ onto the external momenta $k_{i}^{\mu}$,s and eliminating the loop momentum $\ell$ using the scattering equations (3.6). The decomposition of the polarization vector reads,

$$
\epsilon_{i}^{\mu}=\frac{\epsilon\left(\epsilon_{i}, k_{2}, k_{3}, k_{4}\right)}{\epsilon\left(k_{1}, k_{2}, k_{3}, k_{4}\right)} k_{1}^{\mu}+\frac{\epsilon\left(k_{1}, \epsilon_{i}, k_{3}, k_{4}\right)}{\epsilon\left(k_{1}, k_{2}, k_{3}, k_{4}\right)} k_{2}^{\mu}+\frac{\epsilon\left(k_{1}, k_{2}, \epsilon_{i}, k_{4}\right)}{\epsilon\left(k_{1}, k_{2}, k_{3}, k_{4}\right)} k_{3}^{\mu}+\frac{\epsilon\left(k_{1}, k_{2}, k_{3}, \epsilon_{i}\right)}{\epsilon\left(k_{1}, k_{2}, k_{3}, k_{4}\right)} k_{4}^{\mu},
$$

where $\epsilon\left(v_{1}, v_{2}, v_{3}, v_{4}\right)=\epsilon_{\rho_{1} \rho_{2} \rho_{3} \rho_{4}} v_{1}^{\rho_{1}} v_{2}^{\rho_{2}} v_{3}^{\rho_{3}} v_{4}^{\rho_{4}}$ and $\epsilon_{\rho_{1} \rho_{2} \rho_{3} \rho_{4}}$ denotes the Levi-Civita tensor and $v_{i}^{\rho_{i}}$ denotes the $\rho_{i}$-th component of the vector $v_{j}$. Now all the $\epsilon_{i} \cdot \ell$ terms can be rewritten in terms of $\mathcal{R}_{i, j}$ 's using the scattering equations, leading to the following form of (3.9) that treats all the external lines democratically,

$$
\mathcal{F}=\sum_{i \neq j} \frac{\gamma_{[i j]}}{2 \pi i} \mathcal{R}_{i j}
$$

\footnotetext{
${ }^{4} S_{2}$ indicates the permutation of the first two indices. Following cases are similar.
} 
where $\gamma_{[i j]}$ is defined in [47] as

$$
\gamma_{[\rho(1) \rho(2)]}=\langle 12\rangle^{4} \frac{[\rho(1) \rho(2)]^{2}[\rho(3) \rho(4)][\rho(4) \rho(5)][\rho(3) \rho(5)]}{4 \epsilon(\rho(1), \rho(2), \rho(3), \rho(4))},
$$

with $\rho$ being a permutation element in $S_{5} /\left(S_{2} \times S_{3}\right)$.

Substituting (3.11) into (3.1) and taking the limit $\tau \rightarrow \infty$, the one-loop MHV amplitude for five points reads

$$
\mathcal{I}_{5}^{l=1}=\oint \frac{d \sigma_{1} \wedge \cdots \wedge d \sigma_{4}}{f_{1} \cdots f_{4}} \operatorname{PT}_{5}\left(\sum_{i \neq j} \frac{\gamma_{[i j]}}{2} \frac{\sigma_{i}+\sigma_{j}}{\sigma_{i}-\sigma_{j}}\right) \prod_{r=1}^{4} \frac{1}{\sigma_{r}} .
$$

\subsection{Transformations to the prepared CHY integrand}

So far we have reduced the Pfaffian in the SYM integrand to a simple expression. Substituting the explicit expressions for the Parke-Taylor factor and applying the linear transformations that turn the original scattering equations to polynomials, we write down the following expressions for the five-point integrand,

$$
\mathcal{I}_{5}^{l=1}=\oint \frac{d \sigma_{1} \wedge \cdots \wedge d \sigma_{4}}{h_{1} \cdots h_{4}} \prod_{s-r \geqslant 2}^{5}\left(\sigma_{r}-\sigma_{s}\right)\left(\sum_{k=1}^{5} \frac{\sigma_{k+1}-\sigma_{k}}{\sigma_{k+1}}\right)\left(\sum_{i \neq j} \frac{\gamma_{[i j]}}{2} \frac{\sigma_{i}+\sigma_{j}}{\sigma_{i}-\sigma_{j}}\right) .
$$

The factor

$$
\left(\sum_{k=1}^{5} \frac{\sigma_{k+1}-\sigma_{k}}{\sigma_{k+1}}\right)\left(\sum_{i \neq j} \frac{\gamma_{[i j]}}{2} \frac{\sigma_{i}+\sigma_{j}}{\sigma_{i}-\sigma_{j}}\right)
$$

is holomorphically equivalent ${ }^{5}$ to

$$
\sum_{i=1}^{5} \beta_{i, i+1, i+2, i+3, i+4} \frac{\sigma_{i}-\sigma_{i-1}}{2 \sigma_{i}}+\sum_{i, i+1} \gamma_{[i, i+1]} \frac{\sigma_{i}}{\sigma_{i}-\sigma_{i+1}}\left(\sum_{j \neq i+1} \frac{\sigma_{j}-\sigma_{j-1}}{\sigma_{j}}\right),
$$

where $\beta_{i_{1} i_{2} i_{3} i_{4} i_{5}}=\sum_{r<s} \gamma_{\left[i_{r} i_{s}\right]}$. The former term above is already of the prepared form (2.13) up to the global residue theorem (which only introduces an overall minus sign to the result), while the latter, although not yet of the form we want, can be easily massaged into one, using the following cross ratio identities derived in [31],

$$
\frac{s_{i i+1}}{\sigma_{i}-\sigma_{i+1}}=\frac{-1}{\left(\sigma_{i+1}-\sigma_{i+4}\right)}\left(\frac{s_{i i+2}\left(\sigma_{i+2}-\sigma_{i+4}\right)}{\sigma_{i}-\sigma_{i+2}}+\frac{s_{i i+3}\left(\sigma_{i+3}-\sigma_{i+4}\right)}{\sigma_{i}-\sigma_{i+3}}+\frac{l_{i}\left(\sigma_{i}-\sigma_{i+4}\right)}{\sigma_{i}}\right)
$$

where $i \in[1,5]$. The denominator on the right-hand side contains the factors $\left(\sigma_{i}-\sigma_{j}\right)$ of non-adjacent pairs $(i j)$ only and these factors are canceled out by the Vandermonde determinant that was introduced by the linear transformations of the scattering equations, leaving the monomial $\sigma_{i}$ in the denominator, which is just the case of the prepared form up to the global residue theorem.

\footnotetext{
${ }^{5}$ If two meromorphic forms differ from each other by a holomorphic form, they are holomorphically equivalent.
} 
Collecting all the prepared forms, the integrand (3.13) is rearranged as follows,

$$
\mathcal{I}_{5}^{l=1}=\oint \frac{d \sigma_{1} \wedge \cdots \wedge d \sigma_{4}}{h_{1} \cdots h_{4}} \prod_{s-r \geqslant 2}^{5}\left(\sigma_{r}-\sigma_{s}\right)\left(\sum_{i=1}^{5} \mathcal{P}_{i}+\sum_{j \neq i+1}^{5} \mathcal{B}_{[i, i+1], j}\right)
$$

where

$$
\mathcal{P}_{i}=\beta_{i, i+1, i+2, i+3, i+4} \frac{\sigma_{i}-\sigma_{i-1}}{2 \sigma_{i}},
$$

and

$$
\mathcal{B}_{[i, i+1], j}=\left(\sum_{r=2}^{3} \frac{s_{i i+r} \sigma_{i}\left(\sigma_{i+r}-\sigma_{i+4}\right)}{s_{i i+1}\left(\sigma_{i+1}-\sigma_{i+4}\right)\left(\sigma_{i+r}-\sigma_{i}\right)}-\frac{l_{i}\left(\sigma_{i}-\sigma_{i+4}\right)}{s_{i i+1}\left(\sigma_{i+1}-\sigma_{i+4}\right)}\right)\left(\frac{\sigma_{j}-\sigma_{j-1}}{\sigma_{j}}\right) .
$$

\subsection{Evaluating the prepared form of the five point integrand}

In order to evaluate (3.14), we first construct the differential operators that capture the residues corresponding to the prepared expressions obtained previously. Without loss of generality, we take the following prepared form with $h_{5}=\sigma_{1}$ as an example to demonstrate the detailed evaluation (all the other terms can be computed the same way),

$$
\mathcal{I}_{5}^{l=1}=\oint_{h_{1}=\cdots=h_{4}=h_{5}=0} \frac{d \sigma_{1} \wedge \cdots \wedge d \sigma_{4} \wedge d \sigma_{5}}{h_{1} \cdots h_{4} h_{5}} \mathcal{H}(\sigma),
$$

where $\mathcal{H}(\sigma)$ is a holomorphic function of the $\sigma_{i}$ 's. The differential operator for this residue is of order 6 and has 210 coefficients $a_{r_{1}, r_{2}, r_{3}, r_{4}, r_{5}}$ to be determined.

We first get rid of those coefficients that are obviously zero. As discussed in the previous sections, these coefficients are $a_{r_{1}, \cdots, r_{5}}$ with $r_{1} \geq 1$ and those with ranks lower than $3 ! 2 ! 1 !=12$, namely, $a_{0,1,1,1,3}, a_{0,1,1,2,2}$, and $a_{0,0,2,2,2}$ (including permutations of the last four indices). The total number of the vanishing coefficients is

$$
C_{8}^{3}+C_{7}^{3}+C_{6}^{3}+C_{5}^{3}+C_{4}^{3}+C_{3}^{3}+C_{4}^{1}+C_{4}^{2}+C_{4}^{1}=140 .
$$

It is easy to observe that the elementary coefficients in this case are $a_{1,1,1,1,2}, a_{0,1,1,2,2}$ and $a_{0,0,1,2,3}$ (including index permutations) and their corresponding tableaux are depicted in figure 7 . Among them, only $a_{0,0,1,2,3}$ (including the permutations of the last four indices) is non-vanishing. Therefore the intersection number constraint $\mathbb{D} \mathcal{J}=24$ yields

$$
12 \sum_{\rho}(-1)^{\operatorname{sgn}(\rho)} a_{0, \rho(0), \rho(1), \rho(2), \rho(3)} \ell_{v_{3}^{(\rho)}} \ell_{v_{2,3}^{(\rho)}} \ell_{v_{1,2,3}^{(\rho)}} \ell_{2,3,4,5}=24
$$

where $\rho$ runs over all permutations of $\{0,1,2,3\}$ and $v^{(\rho)}$ has the same meaning as in (2.20). For this particular case, these non-vanishing elementary coefficients read,

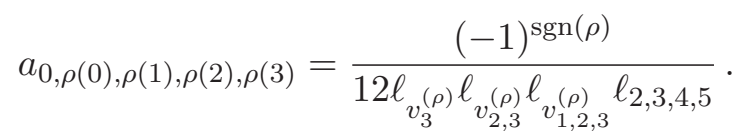



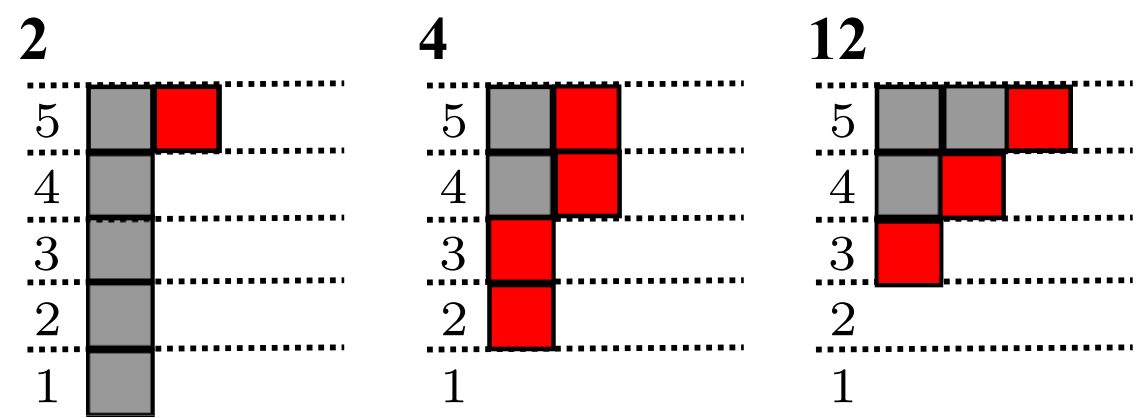

Figure 7. The tableaux for the elementary coefficients: $a_{2,1,1,1,1}, a_{2,2,1,1,0}, a_{3,2,1,0,0}$.

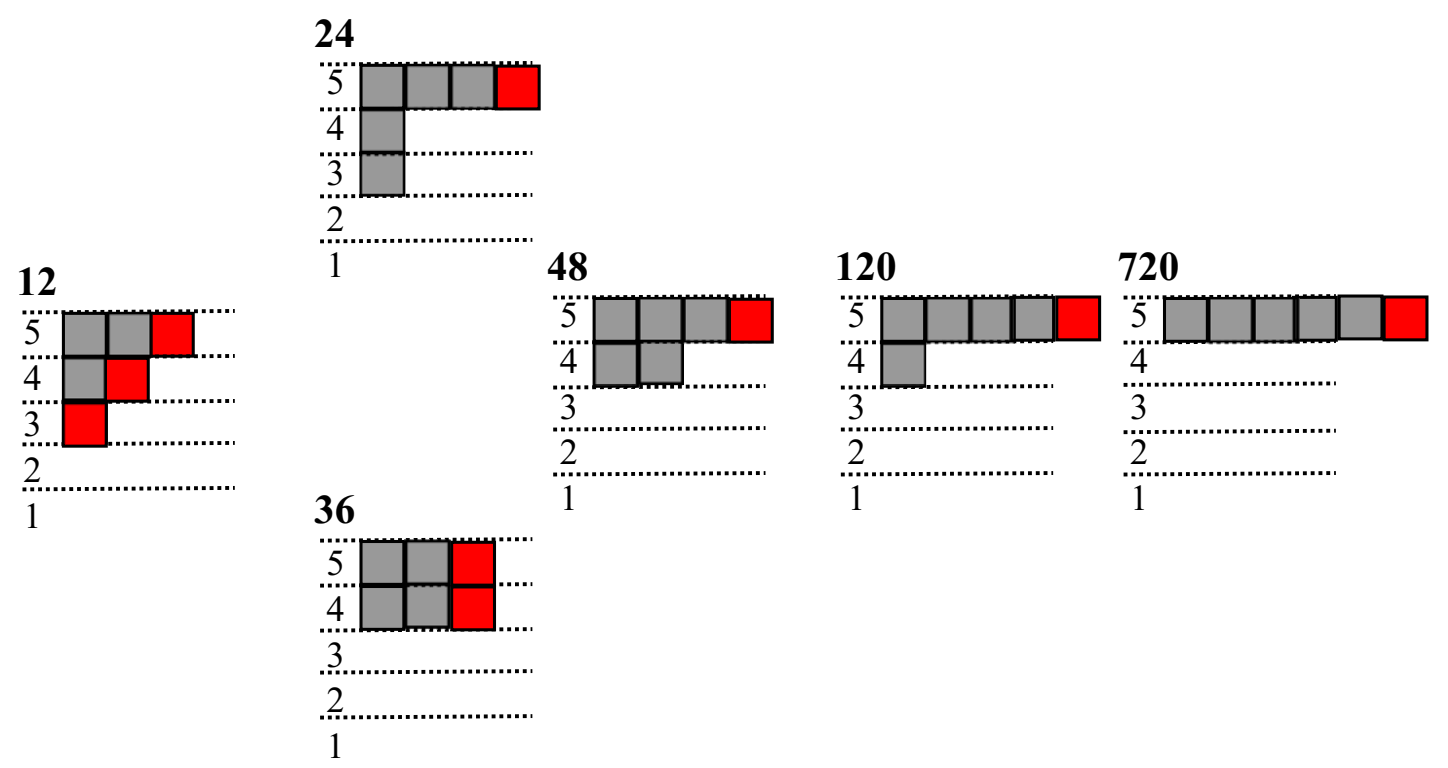

Figure 8. The tableaux of the non-zero $a$ 's for the prepared form in five point amplitude.

Now we are only left with the non-elementary coefficients whose ranks are all higher than 12 , that is, $a_{0,0,1,1,4}, a_{0,0,0,2,2}, a_{0,0,0,2,4}, a_{0,0,0,1,5}$ and $a_{0,0,0,0,6}$. In figure 8 their corresponding tableaux are shown and arranged in such an order that the preferred coloring relates each tableau with only the ones to the left of it. The coefficients corresponding to the two tableaux in the second column in figure 8 , namely $a_{0,0,1,1,4}$ and $a_{0,0,0,3,3}$, can be written in terms of the non-vanishing elementary one alone and the analytic solutions of them read,

$$
\begin{aligned}
a_{0,0,1,1,3+1} & =\frac{-1}{\ell_{5} 4 !}\left[\sum_{1 \leqslant j_{1} \leqslant 5,\left\{j_{1}\right\} \neq\{5\}} \ell_{j_{1}} R(a) a_{r_{1} \cdots\left(r_{j_{1}}+1\right) \cdots r_{5}}\right], \\
a_{0,0,0,2+1,2+1} & =\frac{-1}{3 ! 3 ! \ell_{4,5}}\left[\sum_{\substack{1 \leqslant j_{1}<j_{2} \leqslant 5 \\
\left\{j_{1}, j_{2}\right\} \neq\{4,5\}}} \ell_{j_{1}, j_{2}} R(a) a_{r_{1} \cdots\left(r_{j_{1}}+1\right) \cdots\left(r_{j_{2}}+1\right) \cdots r_{5}}\right],
\end{aligned}
$$




\begin{tabular}{|c|c|c|c|c|}
\hline $\mathcal{P}_{1}$ & $\mathcal{P}_{2}$ & $\mathcal{P}_{3}$ & $\mathcal{P}_{4}$ & $\mathcal{P}_{5}$ \\
\hline$\frac{\beta_{1,2,3,4,5}}{\ell_{5} \ell_{4,5} \ell_{3,4,5} \ell_{2,3,4,5}}$ & $\frac{\beta_{2,3,4,5,1}}{\ell_{1} \ell_{1,5} \ell_{1,4,5} \ell_{1,3,4,5}}$ & $\frac{\beta_{3,4,5,1,2}}{\ell_{2} \ell_{1,2} \ell_{1,2,5} \ell_{1,2,4,5}}$ & $\frac{\beta_{4,5,1,2,3}}{\ell_{3} \ell_{2,3} \ell_{1,2,3} \ell_{1,2,3,5}}$ & $\frac{\beta_{5,1,2,3,4}}{\ell_{4} \ell_{3,4} \ell_{2,3,4} \ell_{1,2,3,4}}$ \\
\hline$E 1$ & $E 2$ & $E 3$ & $E 4$ & $E 5$ \\
\hline
\end{tabular}

Table 1. Final results for the pentagon diagrams. The first line lists the terms while the second line their analytic expressions respectively. The last line shows the forward-limit channels corresponding to each term, where the cut lines in the Q-cut analysis are depicted in figure 9. For a given cut line, its momentum is chosen to be $\ell$.

in which $\vec{r}=(0,0,1,1,3)$ and $(0,0,0,2,2)$ respectively. The coefficients associated with the other three tableaux are worked out in the same fashion and take the following forms,

$$
a_{r_{1}, r_{2}, r_{3}, r_{4}, r_{5}+1}=\frac{-1}{\ell_{5} R\left(a_{r_{1}, r_{2}, r_{3}, r_{4}, r_{5}+1}\right)}\left[\sum_{1 \leqslant j_{1} \leqslant 5,\left\{j_{1}\right\} \neq\{5\}} \ell_{j_{1}} R(a) a_{r_{1} \cdots\left(r_{j_{1}}+1\right) \cdots r_{5}}\right],
$$

where $\vec{r}=(0,0,0,2,3),(0,0,0,1,4),(0,0,0,0,5)$ respectively.

With all the coefficients fixed for the case of (3.17), it is now straightforward to evaluate the $\mathcal{P}_{1}$ and $\mathcal{B}_{[1,2], 1}$ terms in (3.14), by acting with the differential operator $\mathbb{D}$. Explicitly, the $\mathcal{P}_{1}$ term becomes

$$
\begin{aligned}
\mathcal{I}_{5}^{\mathcal{P}_{1}} & =\left.\frac{-\beta_{1,2,3,4,5}}{2} \mathbb{D} \frac{\sigma_{3} \sigma_{4} \sigma_{5}\left(\sigma_{2}-\sigma_{4}\right)\left(\sigma_{2}-\sigma_{5}\right)\left(\sigma_{3}-\sigma_{5}\right)}{\sigma_{5}-1}\right|_{\sigma \rightarrow 0} \\
& =\left.\frac{\beta_{1,2,3,4,5}}{2} \mathbb{D}\left(\sigma_{3} \sigma_{4}^{2} \sigma_{5}^{3}\right)\right|_{\sigma \rightarrow 0} \\
& =3 ! \beta_{1,2,3,4,5} a_{0,0,1,2,3}=\frac{\beta_{1,2,3,4,5}}{2 \ell_{5} \ell_{4,5} \ell_{3,4,5} \ell_{2,3,4,5}} .
\end{aligned}
$$

The integrand term containing $\mathcal{B}_{[1,2], 1}$ is holomorphically equivalent to

$$
\begin{aligned}
\mathcal{I}_{5}^{\mathcal{B}_{[1,2], 1}} & =\left(-\frac{\gamma_{[12]} \ell_{1}}{s_{12}}\right) \oint \frac{d \sigma_{1} \wedge \cdots \wedge d \sigma_{4}}{h_{1} \cdots h_{4} \sigma_{1}} \sigma_{3} \sigma_{4} \sigma_{5}^{2}\left(\sigma_{2}-\sigma_{4}\right)\left(\sigma_{3}-\sigma_{5}\right) \\
& =\left(\frac{\gamma_{[12]} \ell_{1}}{s_{12}}\right) \mathbb{D}\left(\frac{\sigma_{3} \sigma_{4}^{2} \sigma_{5}^{3}}{\sigma_{5}-1}\right)=\left(\frac{\gamma_{[12]} \ell_{1}}{s_{12}}\right) 3 ! 2 ! a_{0,0,1,2,3}=\frac{-\gamma_{[12]}}{s_{12} \ell_{5} \ell_{4,5} \ell_{3,4,5}} .
\end{aligned}
$$

All other terms are similar. The final results for all the terms are shown in table 1 and 2, where the correspondence between each term and a particular forward-limit channel in the Q-cut analysis [48] is displayed.

This concludes the computation of the residues at finite poles and at infinity (the computation of the residue at infinity is transformed to evaluating a prepared form with $h_{n}=\sigma_{n}$ in the same fashion as in [44]).

\subsection{Analysis for the spurious poles}

In this subsection we investigate the spurious poles that arise from the extra solutions to the polynomial scattering equations. Such contribution should vanish in consistent supersymmetric theories and we will show this is indeed true in this particular case. It is easy 


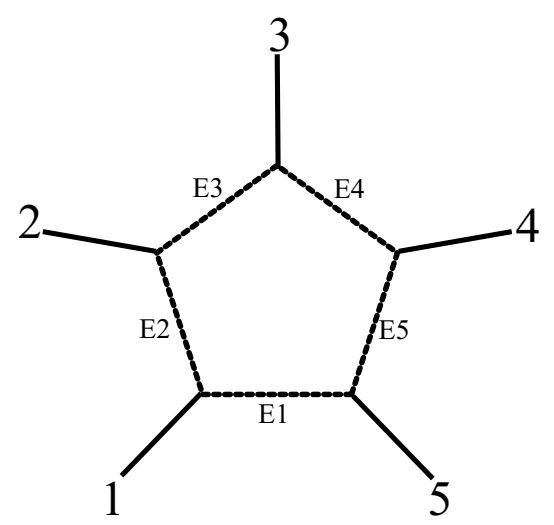

Figure 9. Pentagon diagram.

\begin{tabular}{|c|c|c|c|}
\hline $\mathcal{B}_{[i, i+1], i}$ & $\mathcal{B}_{[i, i+1], i+2}$ & $\mathcal{B}_{[i, i+1], i+3}$ & $\mathcal{B}_{[i, i+1], i+4}$ \\
\hline$\frac{-\gamma_{i, i+1} / s_{i, i+1}}{\ell_{i+4} \ell_{i+3, i+4} \ell_{i+2, i+3, i+4}}$ & $\frac{-\gamma_{i, i+1} / s_{i, i+1}}{\ell_{i+3} \ell_{i+2, i+3} \ell_{i, i+1, i+2, i+3}}$ & $\frac{-\gamma_{i, i+1} / s_{i, i+1}}{\ell_{i+2} \ell_{i, i+1, i+2} \ell_{i, i+1, i+2, i+4}}$ & $\frac{-\gamma_{i, i+1} / s_{i, i+1}}{\ell_{i, i+1} \ell_{i, i+1, i+4} \ell_{i, i+1, i+3, i+4}}$ \\
\hline$E 1$ & $E 3$ & $E 4$ & $E 5$ \\
\hline
\end{tabular}

Table 2. Final results for the box diagrams, $i \in[1,5]$. Like in table 1 , the first and the second lines show the terms and their respective final expressions, while in the last line their corresponding forward-limit channels are given and the cut lines are depicted in figure 10. Again, for a given cut line in the Q-cut analysis, its momentum is chosen to be $\ell$.

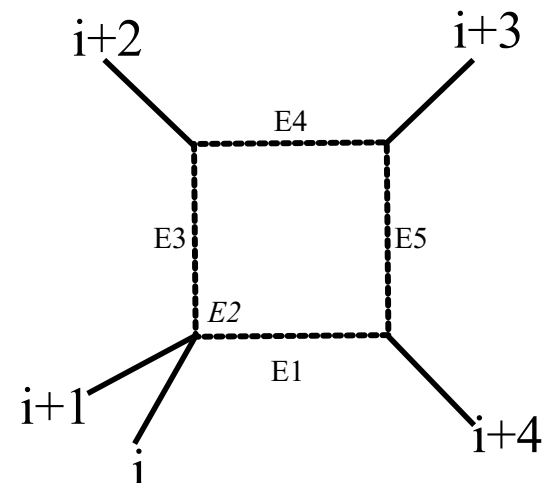

Figure 10. Box diagram.

to observe that the extra solutions all coincide at the position $\sigma_{1}=\sigma_{2}=\cdots=\sigma_{n-1}=1$. Naturally, we prefer to deal with poles at the origin and shift the spurious pole there by the parameter transformation $\sigma_{i} \rightarrow \sigma_{i}+1$. Therefore the residue at the spurious pole is associated with the meromorphic form,

$$
\rho_{\text {spurious }}=\frac{d \sigma_{1} \wedge d \sigma_{2} \wedge d \sigma_{3} \wedge d \sigma_{4}}{h_{1}\left(\sigma_{j}+1\right) h_{2}\left(\sigma_{j}+1\right) h_{3}\left(\sigma_{j}+1\right) h_{4}\left(\sigma_{j}+1\right)} \mathcal{H}_{5}\left(\sigma_{j}+1\right)
$$


where the factor $\mathcal{H}_{5}$ is read off from (3.14) and

$$
\mathcal{H}_{5}\left(\sigma_{j}+1\right)=\sum_{r<s} \beta_{[r s]} \sum_{\rho \in S_{5}} \frac{\prod_{i<j}^{5}\left(\sigma_{i}-\sigma_{j}\right)}{\left(\sigma_{\rho(1)}+1\right)\left(\sigma_{\rho(1)}-\sigma_{\rho(2)}\right) \cdots\left(\sigma_{\rho(4)}-\sigma_{\rho(5)}\right)} \frac{\sigma_{r}+\sigma_{s}+2}{\sigma_{r}-\sigma_{s}} .
$$

The product $\prod_{i+1<j}\left(\sigma_{i}-\sigma_{j}\right)$ contains all non-adjacent pairs of $\left(\sigma_{i}-\sigma_{j}\right)$ with the gauge choice being implicitly $\sigma_{5}=1$. The shifted polynomial scattering equations read the following,

$$
\begin{aligned}
h_{1}\left(\sigma_{j}+1\right)= & \sum_{i=1}^{5} \ell_{i}+\sum_{i=1}^{4} \ell_{i} \sigma_{i}, \\
h_{2}\left(\sigma_{j}+1\right)= & -\sum_{i_{1}<i_{2}}^{5} \ell_{i_{1}, i_{2}}-\sum_{i_{1}=1}^{4} \sum_{i_{2} \neq i_{1}}^{5} \ell_{i_{1}, i_{2}} \sigma_{i_{1}}-\sum_{i_{1}<i_{2}}^{4} \ell_{i_{1}, i_{2}} \sigma_{i_{1}} \sigma_{i_{2}}, \\
h_{3}\left(\sigma_{j}+1\right)= & \sum_{i_{1}<i_{2}<i_{3}}^{5} \ell_{i_{1}, i_{2}, i_{3}}+\sum_{i_{1}=1}^{4} \sum_{i_{2}<i_{3} \neq i_{1}}^{5} \ell_{i_{1}, i_{2}, i_{3}} \sigma_{i_{1}}+\sum_{i_{1}<i_{2}}^{4} \sum_{i_{3} \neq i_{1}, i_{2}}^{5} \ell_{i_{1}, i_{2}, i_{3}} \sigma_{i_{1}} \sigma_{i_{2}} \\
& +\sum_{i_{1}<i_{2}<i_{3}}^{4} \ell_{i_{1}, i_{2}, i_{3}} \sigma_{i_{1}} \sigma_{i_{2}} \sigma_{i_{3}}, \\
h_{4}\left(\sigma_{j}+1\right)= & -\sum_{i_{1}<\cdots<i_{4}}^{5} \ell_{i_{1}, i_{2}, i_{3}, i_{4}}-\sum_{i_{1}=1}^{4} \sum_{i_{2}<i_{3}<i_{4} \neq i_{1}}^{5} \ell_{i_{1}, i_{2}, i_{3}, i_{4}} \sigma_{i_{1}}-\sum_{i_{1}<i_{2}}^{4} \sum_{i_{3}<i_{4} \neq i_{1}, i_{2}}^{5} \ell_{i_{1}, i_{2}, i_{3}, i_{4}} \sigma_{i_{1}} \sigma_{i_{2}} \\
& -\sum_{i_{1}<i_{2}<i_{3}}^{4} \sum_{i_{4} \neq i_{1}, i_{2} i_{3}}^{5} \ell_{i_{1}, i_{2}, i_{3}, i_{4}} \sigma_{i_{1}} \sigma_{i_{2}} \sigma_{i_{3}}-\sum_{i_{1}<\cdots i_{4}}^{4} \ell_{i_{1}, i_{2}, i_{3}, i_{4}} \sigma_{i_{1}} \sigma_{i_{2}} \sigma_{i_{3}} \sigma_{i_{4}} .
\end{aligned}
$$

Note that the leading order terms above do not give rise to a zero-dimensional intersection and we have to apply transformations on these polynomials such that the divisors generated by them coincide at an isolated point. The explicit expressions for such transformations will not be needed and we simply display the new polynomials here,

$$
\begin{aligned}
& \hat{h}_{1}=\sum_{i=1}^{4} \ell_{i} \sigma_{i}, \\
& \hat{h}_{2}=-\sum_{i_{1}<i_{2}}^{4} \ell_{i_{1}, i_{2}} \sigma_{i_{1}} \sigma_{i_{2}}, \\
& \hat{h}_{3}=\sum_{i_{1}<i_{2}}^{4} \sum_{i_{3} \neq i_{1}, i_{2}}^{5} \ell_{i_{1}, i_{2}, i_{3}} \sigma_{i_{1}} \sigma_{i_{2}}+\sum_{i_{1}<i_{2}<i_{3}}^{4} \ell_{i_{1}, i_{2}, i_{3}} \sigma_{i_{1}} \sigma_{i_{2}} \sigma_{i_{3}}, \\
& \hat{h}_{4}=-\left(\sum_{i_{1}<i_{2}<i_{3}}^{4} \sum_{i_{4} \neq i_{1}, i_{2}, i_{3}}^{5} \ell_{i_{1}, i_{2}, i_{3}, i_{4}} \sigma_{i_{1}} \sigma_{i_{2}} \sigma_{i_{3}}+\sum_{i_{1}<\cdots i_{4}}^{4} \ell_{i_{1}, i_{2}, i_{3}, i_{4}} \sigma_{i_{1}} \sigma_{i_{2}} \sigma_{i_{3}} \sigma_{i_{4}}\right) .
\end{aligned}
$$

According to the transformation law, we also need to take care of the determinant of the transformations, which is simply 1 here.

In principle we need to construct a different differential operator following section 4.4 of [44]; and to this end the hatted polynomials are homogenized in a way such that the 
higher-order terms are lowered to the same degree as the leading order ones, leading to the order of this operator being 4 . Luckily the actual form of the operator is not necessary in this case, since (3.24) is holomorphic at the shifted origin and the lowest term in its numerator is of degree 5. The action of the fourth-order differential operator on (3.24) must vanish when evaluated at the shifted origin.

Since the spurious poles do not contribute, the total residue obtained previously is the final integrand for the five-point SYM amplitude at one loop which simply reads,

$$
\mathcal{I}_{5}^{l=1}=\sum_{i=1}^{5} \mathcal{I}_{5}^{\mathcal{P}_{i}}+\sum_{j \neq i+1}^{5} \mathcal{I}_{5}^{\mathcal{B}_{[i, i+1], j}}
$$

where the expressions for $\mathcal{I}_{5}^{\mathcal{P}_{i}}$ and $\mathcal{I}_{5}^{\mathcal{B}_{[i, i+1], j}}$ are given in table 1 and table 2. Just like its fourpoint counterpart studied in [44], our result presents a clear one-to-one correspondence with the forward-limit channels in the Q-cut analysis. We expect this nice property continues to hold for higher points.

The method we have illustrated so far generalizes naturally to the one-loop integrands for any number of external particles. For higher-point cases, we expect the integrands can be decomposed into several residues that are associated with the prepared form, or similar expressions with slightly more complicated $h_{n}$ 's.

Similar to the analysis in section 2 , when $h_{n}$ is a monomial, the corresponding local duality theorem leads to the vanishing of some coefficients.

The local duality equations arising from the scattering equations are universal despite the choice of $h_{n}$, that is to say, the fact that the coefficients in the corresponding differential operator can be related to those with lower or equal ranks only holds for any number of points, and most of the relations among these coefficients remain the same. The construction of the differential operator always boils down to solving for the elementary coefficients. Since the number of the elementary coefficients is quite limited, these coefficients can be solved efficiently.

\section{One-loop $n$-gon amplitude: a direct evaluation}

In this section we discuss the evaluation of the generalized CHY form for one-loop $n$-gon amplitude. The evaluation can be done directly since all $n$-gon amplitudes are already of the prepared form. The one-loop $n$-gon amplitude is conjectured to be the following in [17]

$$
\mathcal{I}_{n}=\oint \frac{d \sigma_{1} \cdots d \sigma_{n-1}}{h_{1} \cdots h_{n-1}} \mathrm{PT}_{n} \prod_{i<j}^{n}\left(\sigma_{i}-\sigma_{j}\right),
$$

where $\mathrm{PT}_{4}$ is the Parke-Taylor factor. The terms in (4.1), after homogenization, are of form

$$
\mathcal{I}_{n}=\sum_{\rho \in \mathcal{S}_{n}} \mathcal{I}_{n}^{\rho(1)}
$$

where

$$
\mathcal{I}_{n}^{\rho(1)}=\oint \frac{d \sigma_{1} \wedge \cdots \wedge d \sigma_{n}}{\tilde{h}_{1} \cdots \tilde{h}_{n-1} \sigma_{\rho(1)}} \sigma_{\rho(n)} \prod_{i \| j}^{n}\left(\sigma_{\rho(i)}-\sigma_{\rho(j)}\right),
$$


where $i \| j$ denotes pairs of neighboring indices in the cyclic order. In terms of the differential operator representation, we have

$$
\begin{aligned}
\mathcal{I}_{n}^{\rho(1)} & =-\left.\mathbb{D} \frac{-\sigma_{\rho(n)} \prod_{i \| j}^{n}\left(\sigma_{\rho(i)}-\sigma_{\rho(j)}\right)}{\sigma_{\rho(1)}-1}\right|_{\sigma \rightarrow 0} \\
& =\mathbb{D} \frac{\sigma_{\rho(3)}^{1} \sigma_{\rho(4)}^{2} \cdots \sigma_{\rho(n)}^{n-2}}{1-\sigma_{\rho(1)}} \\
& =a_{v_{1}^{(\rho-1)}, v_{2}^{(\rho-1)}, \cdots, v_{n}^{(\rho-1)}},
\end{aligned}
$$

where $v^{\left(\rho^{-1}\right)}$ means the result of performing $\rho^{-1}$ permutation on $v=\{0,0,1,2,3, \cdots$, $n-3, n-2\}$, and subscripts of $v$ indexes the components of $v^{\left(\rho^{-1}\right)}$. These $a$ 's have already been obtained in eq. (2.29). On the other hand, as we will see from later discussion, here the spurious pole does not contribute. Thus the sum over $\rho \in \mathcal{S}_{n}$ of (4.3) is the final result of the general one-loop $n$-gon amplitudes.

The analysis for the spurious pole. Here we show that in this case there is indeed no contribution from the spurious pole, which is located at $\sigma_{1}=\cdots=\sigma_{n-1}=1$. The parameter transformation $\sigma_{j} \rightarrow \sigma_{j}+1$ shifts the pole to the origin. The polynomial scattering equations are those appearing in (2.4). Therefore, what we want to compute here is the residue at the origin of the differential form,

$$
\Gamma_{\text {spurious }}=\frac{d \sigma_{1} \wedge d \sigma_{2} \cdots \wedge d \sigma_{n-1}}{h_{1}\left(\sigma_{j}+1\right) h_{2}\left(\sigma_{j}+1\right) \cdots h_{n-1}\left(\sigma_{j}+1\right)} \mathcal{H}_{n}\left(\sigma_{j}+1\right),
$$

where

$$
h_{m}\left(\sigma_{j}+1\right)=(-)^{m+1} \sum_{r=0}^{m} \sum_{i_{1}<\cdots<i_{r}}^{n-1} \sum_{\substack{i_{r+1}<\cdots<i_{m} \\ \neq i_{1}, \cdots, i_{r}}}^{n} \ell_{i_{1}, \cdots, i_{m}} \sigma_{i_{1}} \sigma_{i_{2}} \cdots \sigma_{i_{r}} .
$$

For instance, the first three of the scattering equations are explicitly

$$
\begin{aligned}
h_{1}\left(\sigma_{j}+1\right)= & \sum_{i=1}^{n} \ell_{i}+\sum_{i=1}^{n-1} \ell_{i} \sigma_{i}, \\
h_{2}\left(\sigma_{j}+1\right)= & -\left(\sum_{i_{1}<i_{2}}^{n} \ell_{i_{1}, i_{2}}+\sum_{i_{1}=1}^{n-1} \sum_{i_{2} \neq i_{1}}^{n} \ell_{i_{1}, i_{2}} \sigma_{i_{1}}+\sum_{i_{1}<i_{2}}^{n-1} \ell_{i_{1}, i_{2}} \sigma_{i_{1}} \sigma_{i_{2}}\right), \\
h_{3}\left(\sigma_{j}+1\right)= & \sum_{i_{1}<i_{2}<i_{3}}^{n} \ell_{i_{1}, i_{2}, i_{3}}+\sum_{i_{1}=1}^{n-1} \sum_{i_{2}<i_{3} \neq i_{1}}^{n} \ell_{i_{1}, i_{2}, i_{3}} \sigma_{i_{1}}+\sum_{i_{1}<i_{2}}^{n-1} \sum_{i_{3} \neq i_{1}}^{n} \ell_{i_{1}, i_{2}, i_{3}} \sigma_{i_{1}} \sigma_{i_{2}} \\
& +\sum_{i_{1}<i_{2}<i_{3}}^{n-1} \ell_{i_{1}, i_{2}, i_{3}} \sigma_{i_{1}} \sigma_{i_{2}} \sigma_{i_{3}} .
\end{aligned}
$$

The leading terms of these polynomials form a set of equations in $\sigma$ 's and according to the momentum conservation and on-shell conditions, the solutions to these equations are not zero-dimensional. For such cases, we need to perform a transformation law, then we have

$$
\Gamma_{\text {spurious }}=\frac{d \sigma_{1} \wedge d \sigma_{2} \cdots \wedge d \sigma_{n-1}}{\hat{h}_{1} \hat{h}_{2} \cdots \hat{h}_{n-1}} \mathcal{H}_{n}\left(\sigma_{j}+1\right)
$$


where the polynomials become

$$
\begin{aligned}
\hat{h}_{1} & =\sum_{i=1}^{n-1} \ell_{i} \sigma_{i}, \\
\hat{h}_{2} & =-\sum_{i_{1}<i_{2}}^{n-1} \ell_{i_{1}, i_{2}} \sigma_{i_{1}} \sigma_{i_{2}}, \\
\hat{h}_{m} & =(-)^{m+1} \sum_{r=m-1}^{m} \sum_{i_{1}<\cdots<i_{r}}^{n-1} \sum_{\substack{i_{r+1}<\cdots<i_{m} \\
\neq i_{1}, \cdots, i_{r}}}^{n} \ell_{i_{1}, \cdots, i_{m}} \sigma_{i_{1}} \sigma_{i_{2}} \cdots \sigma_{i_{r}}, m \in[3, n-1] .
\end{aligned}
$$

The intersection number of $\hat{h}_{m}$ at shifted origin is $2(n-2)$ !. This indicates that there are $2(n-2)$ ! solutions of $\sigma$ concentrating on the spurious point. The residue on the shifted origin is well-defined only when $\mathcal{H}_{n}\left(\sigma_{j}+1\right)$ is holomorphic at the origin. For $n$-gon,

$$
\mathcal{H}_{n}\left(\sigma_{j}+1\right)=\left.\sum_{\rho \in S_{n}} \frac{\prod_{i<j}^{n}\left(\sigma_{i}-\sigma_{j}\right)}{\left(\sigma_{\rho(1)}+1\right)\left(\sigma_{\rho(1)}-\sigma_{\rho(2)}\right)\left(\sigma_{\rho(2)}-\sigma_{\rho(3)}\right) \cdots\left(\sigma_{\rho(n-1)}-\sigma_{\rho(n)}\right)}\right|_{\sigma_{n} \rightarrow 0} .
$$

It is easy to see that each factor $\sigma_{\rho(i)}-\sigma_{\rho(i+1)}$ in the dominator will be canceled by a factor in the numerator. The finial degree of $\sigma$ in the numerator is

$$
\frac{(n-2)(n-1)}{2}
$$

According to our conjecture, the differential operator $\mathbb{D}$ for the residue at the shifted origin is

$$
\frac{(n-4)(n-1)}{2}+2
$$

where $n \geqslant 4$. Then it is easy to find that the spurious residues are all vanishing for $n$-gon with $n \geqslant 4$.

\section{Conclusion and outlook}

In this paper we use our previously proposed differential operator to compute the residues on the solutions of scattering equations. We find that the coefficients in the differential operator can be determined in a combinatoric way. We present an analytical solution for the differential operator for the prepared forms of the generalized CHY integrands for $n$ point scattering amplitudes. We then use said differential operator to evaluate the one-loop CHY integrand for five points in $\mathcal{N}=4 \mathrm{SYM}$ which is casted into the prepared forms, and the one-loop CHY integrands of the $n$-gon amplitudes which are naturally of the prepared forms for any number of external lines.

In both examples, we arrive at the compact final expression effortlessly using our method and the final results are analytical and identical with the ones obtained through the Q-cut analysis respectively. Although the examples studied in this paper can be massaged to the prepared form, more complicated denominators may turn up in the integrands when we investigate higher-point one-loop amplitudes in super Yang-Mills. An immediate 
followup is to generalize our method for differential operators corresponding to these new ingredients and determine how efficient this method can be for higher-point amplitudes in comparison with other methods. We are hopeful that it is possible to work out such generalizations and solve for the corresponding differential operator analytically, which will lead to a straightforward evaluation of the one-loop generalized CHY forms.

At the moment of writing, the construction of generalized CHY forms in super YangMills theory has been promoted to two loops for four external lines. Another natural direction to take is to study the combinatoric structures of the two-loop scattering equations and lift the coefficient-generating method to two loops, which in turn will provide a useful tool in constructing the generalized CHY form for any number of external lines at two-loop level.

Moreover, the method we propose is theory-independent and thus has the potential to evaluate any CHY-type expressions in pure Yang-Mills and Gravity theory. This, in turn, will make the generalized CHY forms an efficient method for computing amplitudes.

\section{Acknowledgments}

G.C. and T.W. thank E.Y. Yuan, Y. Zhang, R.J. Huang, B. Feng and H. Johansson for useful discussion and kind suggestions. The research of G.C. is supported in part by the Knut and Alice Wallenberg Foundation under grant KAW 2013.0235, and the Ragnar Söderberg Foundation under grant S1/16. G.C. has been supported in parts by NSF of China Grant under Contract 11405084, the Open Project Program of State Key Laboratory of Theoretical Physics, Institute of Theoretical Physics, Chinese Academy of Sciences (No. Y5KF171CJ1). Y.-K.E.C. acknowledges the European Union's Horizon 2020 research and innovation programme under the Marie Sklodowska-Curie grant agreement No. 644121, and the Priority Academic Program Development for Jiangsu Higher Education Institutions (PAPD).

Open Access. This article is distributed under the terms of the Creative Commons Attribution License (CC-BY 4.0), which permits any use, distribution and reproduction in any medium, provided the original author(s) and source are credited.

\section{References}

[1] D.J. Gross and J.L. Manes, The high-energy behavior of open string scattering, Nucl. Phys. B 326 (1989) 73 [inSPIRE].

[2] P. Caputa and S. Hirano, Observations on open and closed string scattering amplitudes at high energies, JHEP 02 (2012) 111 [arXiv:1108.2381] [INSPIRE].

[3] E. Witten, Parity invariance for strings in twistor space, Adv. Theor. Math. Phys. 8 (2004) 779 [hep-th/0403199] [InSPIRE].

[4] F. Cachazo, Fundamental BCJ relation in $\mathcal{N}=4 S Y M$ from the connected formulation, arXiv:1206.5970 [INSPIRE].

[5] F. Cachazo, S. He and E.Y. Yuan, Scattering of massless particles in arbitrary dimensions, Phys. Rev. Lett. 113 (2014) 171601 [arXiv:1307.2199] [INSPIRE]. 
[6] F. Cachazo, S. He and E.Y. Yuan, Scattering of massless particles: scalars, gluons and gravitons, JHEP 07 (2014) 033 [arXiv:1309.0885] [INSPIRE].

[7] F. Cachazo, S. He and E.Y. Yuan, Einstein-Yang-Mills scattering amplitudes from scattering equations, JHEP 01 (2015) 121 [arXiv:1409.8256] [INSPIRE].

[8] L. Dolan and P. Goddard, Proof of the formula of Cachazo, He and Yuan for Yang-Mills tree amplitudes in arbitrary dimension, JHEP 05 (2014) 010 [arXiv: 1311.5200] [INSPIRE].

[9] L. Dolan and P. Goddard, The polynomial form of the scattering equations, JHEP 07 (2014) 029 [arXiv: 1402.7374] [INSPIRE].

[10] N. Berkovits, Infinite tension limit of the pure spinor superstring, JHEP 03 (2014) 017 [arXiv: 1311.4156] [INSPIRE].

[11] L. Mason and D. Skinner, Ambitwistor strings and the scattering equations, JHEP 07 (2014) 048 [arXiv: 1311.2564] [INSPIRE].

[12] Y. Geyer, A.E. Lipstein and L.J. Mason, Ambitwistor strings in four dimensions, Phys. Rev. Lett. 113 (2014) 081602 [arXiv:1404.6219] [INSPIRE].

[13] E. Casali, Y. Geyer, L. Mason, R. Monteiro and K.A. Roehrig, New ambitwistor string theories, JHEP 11 (2015) 038 [arXiv: 1506.08771] [INSPIRE].

[14] Y. Geyer, L. Mason, R. Monteiro and P. Tourkine, Loop integrands for scattering amplitudes from the Riemann sphere, Phys. Rev. Lett. 115 (2015) 121603 [arXiv:1507.00321] [INSPIRE].

[15] Y. Geyer, L. Mason, R. Monteiro and P. Tourkine, One-loop amplitudes on the Riemann sphere, JHEP 03 (2016) 114 [arXiv:1511.06315] [INSPIRE].

[16] Y. Geyer, L. Mason, R. Monteiro and P. Tourkine, Two-loop scattering amplitudes from the Riemann sphere, Phys. Rev. D 94 (2016) 125029 [arXiv:1607.08887] [INSPIRE].

[17] F. Cachazo, S. He and E.Y. Yuan, One-loop corrections from higher dimensional tree amplitudes, JHEP 08 (2016) 008 [arXiv: 1512.05001] [INSPIRE].

[18] S. He and E.Y. Yuan, One-loop scattering equations and amplitudes from forward limit, Phys. Rev. D 92 (2015) 105004 [arXiv:1508.06027] [INSPIRE].

[19] B. Feng, CHY-construction of planar loop integrands of cubic scalar theory, JHEP 05 (2016) 061 [arXiv:1601.05864] [INSPIRE].

[20] F. Cachazo and H. Gomez, Computation of contour integrals on $\mathcal{M}_{0, n}$, JHEP 04 (2016) 108 [arXiv: 1505.03571] [INSPIRE].

[21] H. Gomez, $\Lambda$ scattering equations, JHEP 06 (2016) 101 [arXiv: 1604.05373] [INSPIRE].

[22] C. Cardona and H. Gomez, Elliptic scattering equations, JHEP 06 (2016) 094 [arXiv: 1605.01446] [INSPIRE].

[23] H. Gomez, S. Mizera and G. Zhang, CHY loop integrands from holomorphic forms, JHEP 03 (2017) 092 [arXiv: 1612.06854] [INSPIRE].

[24] C. Baadsgaard, N.E.J. Bjerrum-Bohr, J.L. Bourjaily and P.H. Damgaard, Integration rules for scattering equations, JHEP 09 (2015) 129 [arXiv:1506.06137] [INSPIRE].

[25] C.S. Lam and Y.-P. Yao, Role of Möbius constants and scattering functions in Cachazo-He-Yuan scalar amplitudes, Phys. Rev. D 93 (2016) 105004 [arXiv:1512.05387] [INSPIRE]. 
[26] C.S. Lam and Y.-P. Yao, Evaluation of the Cachazo-He-Yuan gauge amplitude, Phys. Rev. D 93 (2016) 105008 [arXiv: 1602.06419] [INSPIRE].

[27] C. Baadsgaard, N.E.J. Bjerrum-Bohr, J.L. Bourjaily, P.H. Damgaard and B. Feng, Integration rules for loop scattering equations, JHEP 11 (2015) 080 [arXiv:1508.03627] [INSPIRE].

[28] C.R. Mafra, Berends-Giele recursion for double-color-ordered amplitudes, JHEP 07 (2016) 080 [arXiv: 1603.09731] [INSPIRE].

[29] R. Huang, B. Feng, M.-x. Luo and C.-J. Zhu, Feynman rules of higher-order poles in CHY construction, JHEP 06 (2016) 013 [arXiv: 1604.07314] [INSPIRE].

[30] N.E.J. Bjerrum-Bohr, J.L. Bourjaily, P.H. Damgaard and B. Feng, Analytic representations of Yang-Mills amplitudes, Nucl. Phys. B 913 (2016) 964 [arXiv:1605.06501] [INSPIRE].

[31] C. Cardona, B. Feng, H. Gomez and R. Huang, Cross-ratio identities and higher-order poles of CHY-integrand, JHEP 09 (2016) 133 [arXiv:1606.00670] [INSPIRE].

[32] C. Kalousios, Massless scattering at special kinematics as Jacobi polynomials, J. Phys. A 47 (2014) 215402 [arXiv:1312.7743] [InSPIRE].

[33] S. Weinzierl, On the solutions of the scattering equations, JHEP 04 (2014) 092 [arXiv: 1402.2516] [INSPIRE].

[34] C.S. Lam, Permutation symmetry of the scattering equations, Phys. Rev. D 91 (2015) 045019 [arXiv: 1410.8184] [INSPIRE].

[35] C. Kalousios, Scattering equations, generating functions and all massless five point tree amplitudes, JHEP 05 (2015) 054 [arXiv: 1502.07711] [INSPIRE].

[36] C. Cardona and C. Kalousios, Comments on the evaluation of massless scattering, JHEP 01 (2016) 178 [arXiv:1509.08908] [INSPIRE].

[37] C. Cardona and C. Kalousios, Elimination and recursions in the scattering equations, Phys. Lett. B 756 (2016) 180 [arXiv:1511.05915] [InSPIRE].

[38] L. Dolan and P. Goddard, General solution of the scattering equations, JHEP 10 (2016) 149 [arXiv: 1511.09441] [INSPIRE].

[39] Y.-j. Du, F. Teng and Y.-s. Wu, CHY formula and MHV amplitudes, JHEP 05 (2016) 086 [arXiv: 1603.08158] [INSPIRE].

[40] R. Huang, J. Rao, B. Feng and Y.-H. He, An algebraic approach to the scattering equations, JHEP 12 (2015) 056 [arXiv:1509.04483] [INSPIRE].

[41] M. Søgaard and Y. Zhang, Scattering equations and global duality of residues, Phys. Rev. D 93 (2016) 105009 [arXiv: 1509.08897] [INSPIRE].

[42] J. Bosma, M. Søgaard and Y. Zhang, The polynomial form of the scattering equations is an H-basis, Phys. Rev. D 94 (2016) 041701 [arXiv:1605.08431] [INSPIRE].

[43] M. Zlotnikov, Polynomial reduction and evaluation of tree- and loop-level CHY amplitudes, JHEP 08 (2016) 143 [arXiv: 1605.08758] [INSPIRE].

[44] T. Wang, G. Chen, Y.-K.E. Cheung and F. Xu, A differential operator for integrating one-loop scattering equations, JHEP 01 (2017) 028 [arXiv:1609.07621] [INSPIRE].

[45] R. Hartshorne, Algebraic geometry, vol. 52, Springer Science \& Business Media (2013).

[46] P. Griffiths and J. Harris, Principles of algebraic geometry, John Wiley \& Sons (2014). 
[47] J.J. Carrasco and H. Johansson, Five-point amplitudes in $\mathcal{N}=4$ super-Yang-Mills theory and $\mathcal{N}=8$ supergravity, Phys. Rev. D 85 (2012) 025006 [arXiv:1106.4711] [INSPIRE].

[48] C. Baadsgaard et al., New representations of the perturbative S-matrix, Phys. Rev. Lett. 116 (2016) 061601 [arXiv: 1509. 02169] [INSPIRE].

[49] Z. Bern, L.J. Dixon, D.C. Dunbar and D.A. Kosower, One-loop n-point gauge theory amplitudes, unitarity and collinear limits, Nucl. Phys. B 425 (1994) 217 [hep-ph/9403226] [INSPIRE].

[50] J. Broedel, C.R. Mafra, N. Matthes and O. Schlotterer, Elliptic multiple zeta values and one-loop superstring amplitudes, JHEP 07 (2015) 112 [arXiv:1412.5535] [INSPIRE]. 